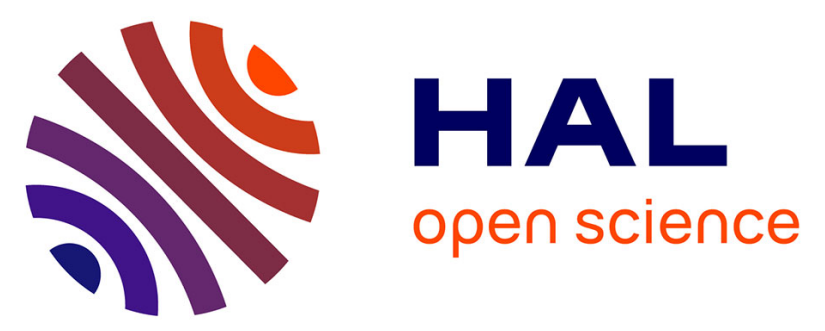

\title{
Pièges de chasse ou dispositifs défensifs? Trois exemples de batteries de fosses à profil en V-Y du Néolithique et de la Protohistoire dans la Marne
}

\author{
Nicolas Garmond, Sophie Binder, Frédéric Poupon
}

\section{- To cite this version:}

Nicolas Garmond, Sophie Binder, Frédéric Poupon. Pièges de chasse ou dispositifs défensifs? Trois exemples de batteries de fosses à profil en V-Y du Néolithique et de la Protohistoire dans la Marne. Bulletin de la Société préhistorique française, 2014, tome 111 (1), pp.53-73. 10.3406/bspf.2014.14364 . hal-02891593

\section{HAL Id: hal-02891593 \\ https://hal.science/hal-02891593}

Submitted on 7 Jul 2020

HAL is a multi-disciplinary open access archive for the deposit and dissemination of scientific research documents, whether they are published or not. The documents may come from teaching and research institutions in France or abroad, or from public or private research centers.
L'archive ouverte pluridisciplinaire HAL, est destinée au dépôt et à la diffusion de documents scientifiques de niveau recherche, publiés ou non, émanant des établissements d'enseignement et de recherche français ou étrangers, des laboratoires publics ou privés. 


\section{Pièges de chasse ou dispositifs défensifs ? Trois exemples de} batteries de fosses à profil en V-Y du Néolithique et de la Protohistoire dans la Marne

Nicolas Garmond, Sophie Binder, Frédéric Poupon

\section{Citer ce document / Cite this document :}

Garmond Nicolas, Binder Sophie, Poupon Frédéric. Pièges de chasse ou dispositifs défensifs ? Trois exemples de batteries de fosses à profil en V-Y du Néolithique et de la Protohistoire dans la Marne. In: Bulletin de la Société préhistorique française, tome 111, $\mathrm{n}^{\circ} 1,2014$. pp. 53-73;

doi : https://doi.org/10.3406/bspf.2014.14364

https://www.persee.fr/doc/bspf_0249-7638_2014_num_111_1_14364

Fichier pdf généré le 21/02/2020 


\section{Abstract}

As a result of recent work in northern France, ' slot pits' have become an important research issue. These pits are large, deep and narrow, with a $\mathrm{V}$ or $\mathrm{Y}$-shaped section. They are often isolated features, containing few artefacts. Although the pits have been interpreted in many ways, three hypotheses can be retained : craft pits, ritual pits or hunting traps. The latter hypothesis is often put forward, in the Champagne-Ardenne region, to explain the recurrent presence in the pits of articulated wild animal skeletons, as well as the absence of contemporary settlements close to the hundred or so pits that have already been recorded. This hypothesis implies an arrangement of the pits in groups. However, in Champagne-Ardennes there are few examples of such groups and most of the pits are isolated features. This article describes three slot-pit groups which were recently discovered in the Marne département (Champagne-Ardenne, France) during preventive archaeological excavations. The first site is the 'Champ de Manoeuvre' at Bétheny, to the north of Reims. During an archaeological evaluation on this site, at some distance from any river, a group of at least ten slot pits was discovered. These features do not display any pattern and their orientation varies. Nevertheless, the group lines up on a broad southwest/ north-east axis. Two of them were investigated : they are quite large, narrow and deep, with many stratified layers visible in section. Two kinds of fill could be distinguished : a silty, homogeneous upper level and a lower part with alternating chalk and silt layers. There were no artefacts apart from a few pottery sherds discovered on the surface, dating the final fill between the Bronze Age and the later Iron Age. Not far from this site, further north in Bétheny at 'Les Écavés', archaeological evaluation work uncovered another group of at least ten slot pits, located on the plateau slope. These pits are also large and deep, with $\mathrm{V}$ or $\mathrm{Y}$ cross-sections. Two or three kinds of fill were identified in the five excavated pits : a silty and homogeneous upper part, and a lower part alternating chalk and silts. A brown silty layer occasionally fills the base of the pit. The spatial organisation of these features is remarkable. Although the pits are 50 metres apart and vary in orientation, they are all on the same contour line. This west-east axis is followed for at least 500 metres. In other words, the line of pits blocks the slope, with large spaces between each pit. Thus, one can imagine a system of traps on the slope, to catch wild animals passing through the area. However the sampled pits produced no finds to confirm this function. More archaeological evidence is required to confirm the hypothesis. While these two groups of slot pits are difficult to date, similar features from Champagne-Ardenne suggest that they are Neolithic, or Bronze Age in date. Although further excavation is necessary, the current evidence from Bétheny does seem compatible with the hunting hypothesis. At SaintMartin-sur-le-Pré ' Rue des Castors', near Châlons-en-Champagne, another group of slot pits was uncovered during preventive excavations. The seven features have narrow $\mathrm{V}$ or $\mathrm{Y}$ shaped sections and deep, stratified fills. Once again, the fills can be divided into two or three parts. Early Neolithic artefacts were found in five pits. Finds include pottery sherds, wild and domestic fauna, with cervid bones in anatomical connection in one pit, as well as flint artefacts and seeds. One slot pit, located near a longhouse, had been reused as a refuse pit. Two radiocarbon dates were obtained. The cervid bones date to the Early Neolithic, so they are contemporary with the Linear Pottery settlement on the site. Cereal seeds from another slot pit in the group date to the Middle Neolithic. These results imply that the slot pits were dug during the Early Neolithic occupation, and remained partially open during the Middle Neolithic. At this site, the hunting hypothesis can be rejected, not only because of the danger of people and domestic animals from the settlement falling into the pits, but also because of the low probability of wild animals approaching this close to the village. The layout of the slot pits on the northern edge of the village, forming an arc of a circle, could be interpreted as an unusual defensive system. This discovery echoes others sites in the Rhineland and Belgium, where similar pits have been found in Linear Pottery settlements. Comparison of the three sites shows similarity in pit morphology, but flagrant differences in spatial organisation. The slot-pit group at Saint-Martin-sur-le-Pré presents some atypical characteristics that are uncommon in Champagne-Ardennes, in particular the association with a contemporary settlement. These three examples show that the debate on the function of slot pits function is still open, and that their function may well have varied over time. 
sur les «fosses à profil en V-Y ». Ces fosses profondes, étroites, souvent vides de mobilier, font l'objet de diverses interprétations, dont l'hypothèse cynégétique qui est souvent avancée en raison de l'isolement de ces fosses par rapport à tout habitat contemporain. Néanmoins, en Champagne-Ardenne, les exemples de batteries de fosses à profil en $\mathrm{V}-\mathrm{Y}$ sont peu courants, puisque la plupart de ces fosses sont retrouvées isolées. Cet article présente trois groupes de fosses à profil en V-Y qui ont récemment été mis au jour dans la Marne. Le premier est localisé au «Champ de Manoeuvre » à Bétheny, au nord de Reims. Sur ce site, loin des cours d'eau, un groupe d'au moins dix fosses à profil en V-Y a été mis au jour lors d'un diagnostic archéologique préventif. Les fosses sont assez massives, étroites et profondes, avec de multiples comblements stratifiés. Elles ne livrent pas de mobilier datant, si ce n'est quelques tessons protohistoriques en surface qui datent leur comblement final. Un peu plus au nord, lors d'un diagnostic préventif aux «Écavés » à Bétheny, un autre dispositif d'au moins dix fosses a été mis au jour sur une pente assez marquée. Les fosses sont massives et profondes, avec des profils en V-Y caractéristiques. L'organisation spatiale est remarquable puisque les fosses, assez éloignées les unes des autres, s'alignent sur un axe situé sur une même courbe de niveau, que l'on peut suivre sur au moins $500 \mathrm{~m}$ de longueur. Elles ne livrent, au diagnostic, aucun mobilier datant. La datation de ces deux ensembles reste difficile, mais, par comparaison avec d'autres fosses du même type, on peut les présumer néolithiques ou protohistoriques. Dans l'attente de fouilles complémentaires, ces deux dispositifs montrent des caractères compatibles avec une interprétation cynégétique. À Saint-Martin-sur-le-Pré, un autre groupe de fosses à profil en $V-Y$ a été mis au jour lors d'une fouille archéologique préventive. Les sept fosses présentent des profils étroits en $\mathrm{V}$ ou en $Y$, assez profonds, avec des comblements stratifiés. Elles livrent du mobilier attribué au Néolithique ancien : céramique, faune, dont des os de cervidés en connexion anatomique, industrie lithique et graines. Deux datations radiocarbones ont été réalisées. Elles démontrent que ces fosses sont contemporaines d'un village du Néolithique ancien situé à proximité immédiate et étaient encore partiellement ouvertes au Néolithique moyen. Pour ce site, l'hypothèse cynégétique est loin de prédominer. II est tout à fait possible que la batterie de fosses à profil en $\mathrm{V}-\mathrm{Y}$, formant un arc de cercle, constitue un dispositif défensif au sens large, qui serait totalement atypique. Cette découverte renvoie à d'autres sites rhénans et belges, où des fosses similaires ont été mises au jour au sein d'habitats enclos du Rubané. Les comparaisons entre nos trois sites montrent des similitudes certaines dans la morphologie des fosses, mais des différences flagrantes dans leur organisation spatiale. La batterie de Saint-Martin-surle-Pré possède des caractères tout à fait atypiques par rapport à ce qui est actuellement connu en Champagne-Ardenne, notamment la présence d'un habitat présumé contemporain. Ces trois exemples montrent que le débat sur la fonction de ces fosses particulières reste encore largement ouvert et qu'il est possible que cette fonction ait pu évoluer au fil du temps. 


\title{
Pièges de chasse ou dispositifs défensifs?
}

\section{Trois exemples de batteries de fosses à profil en V-Y du Néolithique et de la Protohistoire dans la Marne}

\author{
Nicolas Garmond, Sophie Binder et Frédéric Poupon
}

\begin{abstract}
Résumé : Les recherches récentes dans le Nord de la France ont permis l'émergence d'une problématique sur les « fosses à profil en $\mathrm{V}-\mathrm{Y}$ ». Ces fosses profondes, étroites, souvent vides de mobilier, font l'objet de diverses interprétations, dont l'hypothèse cynégétique qui est souvent avancée en raison de l'isolement de ces fosses par rapport à tout habitat contemporain. Néanmoins, en Champagne-Ardenne, les exemples de batteries de fosses à profil en V-Y sont peu courants, puisque la plupart de ces fosses sont retrouvées isolées. Cet article présente trois groupes de fosses à profil en V-Y qui ont récemment été mis au jour dans la Marne. Le premier est localisé au "Champ de Manœuvre » à Bétheny, au nord de Reims. Sur ce site, loin des cours d'eau, un groupe d'au moins dix fosses à profil en V-Y a été mis au jour lors d'un diagnostic archéologique préventif. Les fosses sont assez massives, étroites et profondes, avec de multiples comblements stratifiés. Elles ne livrent pas de mobilier datant, si ce n'est quelques tessons protohistoriques en surface qui datent leur comblement final. Un peu plus au nord, lors d'un diagnostic préventif aux « Écavés » à Bétheny, un autre dispositif d'au moins dix fosses a été mis au jour sur une pente assez marquée. Les fosses sont massives et profondes, avec des profils en V-Y caractéristiques. L'organisation spatiale est remarquable puisque les fosses, assez éloignées les unes des autres, s'alignent sur un axe situé sur une même courbe de niveau, que l'on peut suivre sur au moins $500 \mathrm{~m}$ de longueur. Elles ne livrent, au diagnostic, aucun mobilier datant. La datation de ces deux ensembles reste difficile, mais, par comparaison avec d'autres fosses du même type, on peut les présumer néolithiques ou protohistoriques. Dans l'attente de fouilles complémentaires, ces deux dispositifs montrent des caractères compatibles avec une interprétation cynégétique. À Saint-Martin-sur-le-Pré, un autre groupe de fosses à profil en V-Y a été mis au jour lors d'une fouille archéologique préventive. Les sept fosses présentent des profils étroits en $\mathrm{V}$ ou en $\mathrm{Y}$, assez profonds, avec des comblements stratifiés. Elles livrent du mobilier attribué au Néolithique ancien : céramique, faune, dont des os de cervidés en connexion anatomique, industrie lithique et graines. Deux datations radiocarbones ont été réalisées. Elles démontrent que ces fosses sont contemporaines d'un village du Néolithique ancien situé à proximité immédiate et étaient encore partiellement ouvertes au Néolithique moyen. Pour ce site, l'hypothèse cynégétique est loin de prédominer. Il est tout à fait possible que la batterie de fosses à profil en V-Y, formant un arc de cercle, constitue un dispositif défensif au sens large, qui serait totalement atypique. Cette découverte renvoie à d'autres sites rhénans et belges, où des fosses similaires ont été mises au jour au sein d'habitats enclos du Rubané. Les comparaisons entre nos trois sites montrent des similitudes certaines dans la morphologie des fosses, mais des différences flagrantes dans leur organisation spatiale. La batterie de Saint-Martin-surle-Pré possède des caractères tout à fait atypiques par rapport à ce qui est actuellement connu en Champagne-Ardenne, notamment la présence d'un habitat présumé contemporain. Ces trois exemples montrent que le débat sur la fonction de ces fosses particulières reste encore largement ouvert et qu'il est possible que cette fonction ait pu évoluer au fil du temps.
\end{abstract}

Mots-clés : Néolithique ancien, Rubané, Protohistoire, fosse à profil en V-Y, chasse, Marne.

Abstract: As a result of recent work in northern France, 'slot pits' have become an important research issue. These pits are
large, deep and narrow, with a V or Y-shaped section. They are often isolated features, containing few artefacts. Although
the pits have been interpreted in many ways, three hypotheses can be retained: craft pits, ritual pits or hunting traps. The
latter hypothesis is often put forward, in the Champagne-Ardenne region, to explain the recurrent presence in the pits
of articulated wild animal skeletons, as well as the absence of contemporary settlements close to the hundred or so pits
that have already been recorded. This hypothesis implies an arrangement of the pits in groups. However, in Champagne-
Ardennes there are few examples of such groups and most of the pits are isolated features. This article describes three
slot-pit groups which were recently discovered in the Marne département (Champagne-Ardenne, France) during preven-
tive archaeological excavations. The first site is the 'Champ de Manœuvre' at Bétheny, to the north of Reims. During an
archaeological evaluation on this site, at some distance from any river, a group of at least ten slot pits was discovered.
These features do not display any pattern and their orientation varies. Nevertheless, the group lines up on a broad south-
west/north-east axis. Two of them were investigated: they are quite large, narrow and deep, with many stratified layers
visible in section. Two kinds of fill could be distinguished: a silty, homogeneous upper level and a lower part with alter-
nating chalk and silt layers. There were no artefacts apart from a few pottery sherds discovered on the surface, dating the 
final fill between the Bronze Age and the later Iron Age. Not far from this site, further north in Bétheny at 'Les Écavés', archaeological evaluation work uncovered another group of at least ten slot pits, located on the plateau slope. These pits are also large and deep, with V or Y cross-sections. Two or three kinds of fill were identified in the five excavated pits: a silty and homogeneous upper part, and a lower part alternating chalk and silts. A brown silty layer occasionally fills the base of the pit. The spatial organisation of these features is remarkable. Although the pits are 50 metres apart and vary in orientation, they are all on the same contour line. This west-east axis is followed for at least 500 metres. In other words, the line of pits blocks the slope, with large spaces between each pit. Thus, one can imagine a system of traps on the slope, to catch wild animals passing through the area. However the sampled pits produced no finds to confirm this function. More archaeological evidence is required to confirm the hypothesis. While these two groups of slot pits are difficult to date, similar features from Champagne-Ardenne suggest that they are Neolithic, or Bronze Age in date. Although further excavation is necessary, the current evidence from Bétheny does seem compatible with the hunting hypothesis. At SaintMartin-sur-le-Pré 'Rue des Castors', near Châlons-en-Champagne, another group of slot pits was uncovered during preventive excavations. The seven features have narrow V or Y shaped sections and deep, stratified fills. Once again, the fills can be divided into two or three parts. Early Neolithic artefacts were found in five pits. Finds include pottery sherds, wild and domestic fauna, with cervid bones in anatomical connection in one pit, as well as flint artefacts and seeds. One slot pit, located near a longhouse, had been reused as a refuse pit. Two radiocarbon dates were obtained. The cervid bones date to the Early Neolithic, so they are contemporary with the Linear Pottery settlement on the site. Cereal seeds from another slot pit in the group date to the Middle Neolithic. These results imply that the slot pits were dug during the Early Neolithic occupation, and remained partially open during the Middle Neolithic. At this site, the hunting hypothesis can be rejected, not only because of the danger of people and domestic animals from the settlement falling into the pits, but also because of the low probability of wild animals approaching this close to the village. The layout of the slot pits on the northern edge of the village, forming an arc of a circle, could be interpreted as an unusual defensive system. This discovery echoes others sites in the Rhineland and Belgium, where similar pits have been found in Linear Pottery settlements. Comparison of the three sites shows similarity in pit morphology, but flagrant differences in spatial organisation. The slot-pit group at Saint-Martin-sur-le-Pré presents some atypical characteristics that are uncommon in Champagne-Ardennes, in particular the association with a contemporary settlement. These three examples show that the debate on the function of slot pits function is still open, and that their function may well have varied over time ${ }^{(1)}$.

Keywords: Early Neolithic, Linear Pottery Culture, Protohistory, Slot Pits, Hunting, Marne.

$\mathrm{L}$ A MULTIPLICATION des diagnostics et des fouilles archéologiques préventives a récemment permis le développement d'une problématique spécifique sur les fosses « à profil en $\mathrm{V}, \mathrm{Y}$ ou $\mathrm{W}$ » (Marcigny et Riquier, 2009). Ces fosses profondes, à profil étroit, souvent isolées et vides de vestiges, ont longtemps été délaissées par la recherche archéologique française. Depuis le début du $\mathrm{XX}^{\mathrm{e}}$ siècle, plusieurs interprétations leur ont été données : fosses artisanales, structures cultuelles ou dispositifs cynégétiques néolithiques et protohistoriques.

Dans la Marne, de récentes opérations menées par le service archéologique de Reims Métropole viennent alimenter le débat sur la fonction de ces fosses (fig. 1). Au nord de Reims, à Bétheny, deux dispositifs comprenant de nombreuses fosses, étendus sur de grandes surfaces, ont été découverts. À Saint-Martin-sur-le-Pré, à l'ouest de Châlonsen-Champagne, une autre batterie de fosses à profil en $\mathrm{V}$-Y a été mise au jour au sein même d'un habitat du Rubané.

Ces découvertes, à la lumière des débats récents, invitent à nous interroger sur les différentes interprétations données aux fosses à profil en V-Y.

\section{LES FOSSES À PROFIL EN V-Y : ÉTAT DE LA QUESTION}

es fosses à profil en V-Y sont attestées dans la littérature archéologique dès le début du $\mathrm{Xx}^{\mathrm{e}}$ siècle où, sur des sites néolithiques de la vallée du Rhin, des fosses appelées Schlitzgruben («fosses en fente ») ont été mises au jour (Paret, 1910). Il s'agit de fosses ovales, assez massives, profondes et étroites. Ces fosses sont souvent repérées sur des sites d'habitat du Rubané et font l'objet d'interprétations très diverses : pièges à gibier, fosses de décantation d'argile, fosses de stockage pour la viande, paravents, cachettes d'artisan, trous de poteau, barrages défensifs, puits sacrificiels... (Marcigny et Riquier, 2009).

Une seconde hypothèse va ensuite prévaloir dans la littérature anglo-saxonne et rhénane pour interpréter les Slits, celle des Tan-Pits, (« fosses de tannage »; Van de Velde, 1973). Sans être démontrée par des analyses scientifiques, elle est néanmoins rapidement adoptée par la communauté archéologique.

Longtemps délaissées par l'archéologie française, ces fosses font depuis plusieurs années l'objet de recherches récentes (Marcigny et Riquier, 2009). La reprise de l'ensemble de la documentation disponible a atteint son apogée avec l'organisation d'une table ronde à Châlons-en-Champagne fin 2010. Cette table ronde (Achard-Corompt et al., 2013) a permis de proposer plusieurs pistes d'interprétation, étayées par la documentation archéologique et des analyses scientifiques poussées (datations radiocarbones, analyses de sols, du mobilier, etc.) sur de nombreuses fosses retrouvées dans divers contextes archéologiques.

Le bilan de 2010 (Achard-Corompt et al., 2010) révèle que ces fosses sont présentes sur une large partie de l'Europe et même du monde. En France, elles sont 

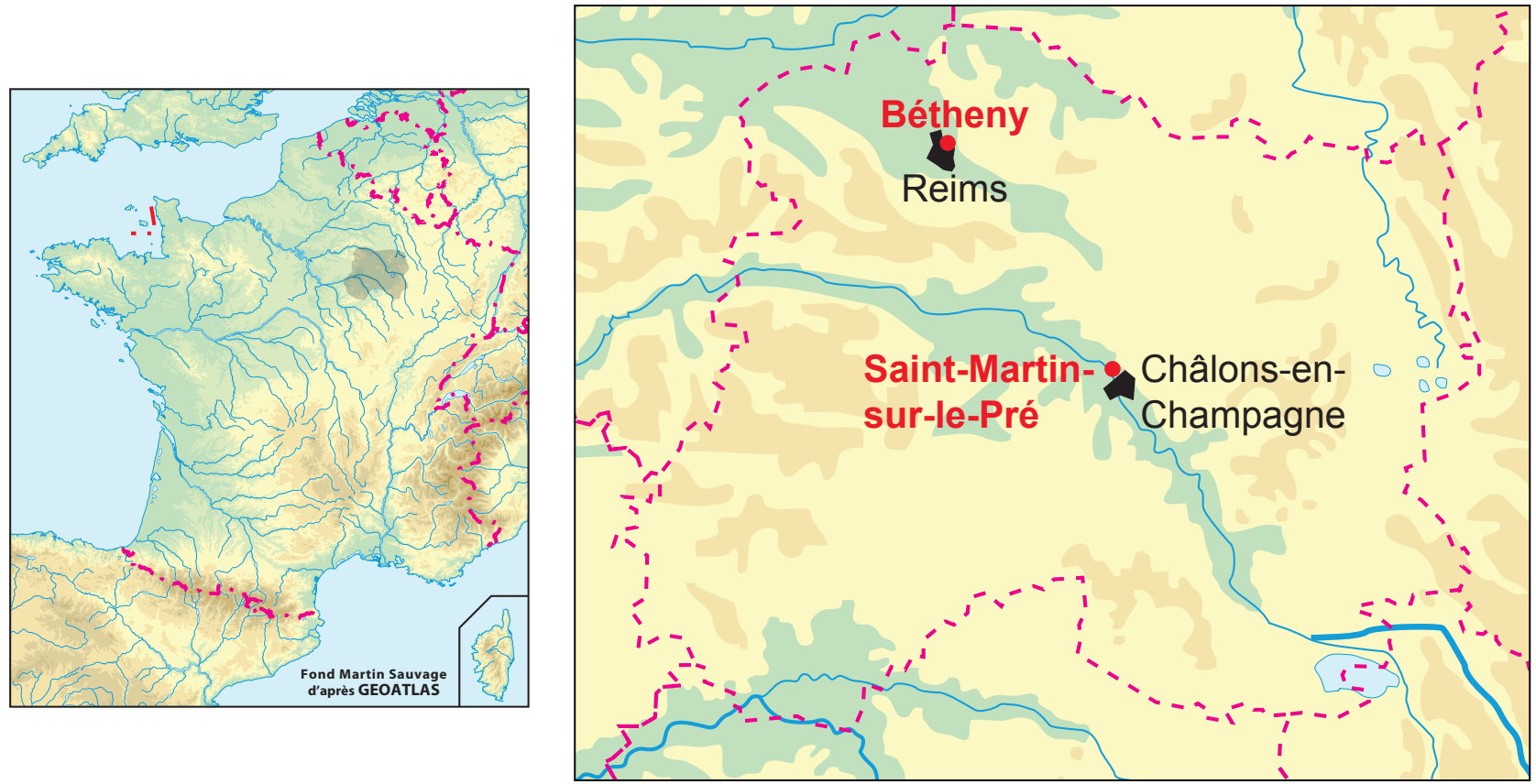

Fig. 1 - Localisation des sites.

Fig. 1 - Location of the sites.

nombreuses (se comptant par centaines en ChampagneArdenne par exemple) et sont retrouvées le plus souvent déconnectées de tout habitat. Ces fosses présentent des profils transversaux variés, en I, U, V voire parfois des profils longitudinaux en W. Elles possèdent des caractéristiques communes : des formes ovales plus ou moins étroites, et une profondeur importante, avec un net rétrécissement du fond. Les comblements sont plutôt limoneux sombres dans les parties sommitales, plutôt proches du substrat (crayeux en Champagne) dans les parties inférieures. La plupart des fosses sont vides de tout mobilier archéologique. Les rares fosses qui en livrent comportent principalement des tessons de céramique et de la faune, avec une relative importance de la faune sauvage.

La réalisation de nombreuses datations radiocarbones sur du mobilier issu de fosses à profil en V-Y révèle qu'en Champagne-Ardenne le "phénomène » se développe entre le Néolithique ancien et l'âge du Fer, avec des pics de fréquence au Néolithique récent et au Bronze final (Achard-Corompt et al., 2010).

Ces constatations ont amené à rejeter les anciennes hypothèses pour se tourner, pour plusieurs chercheurs, vers une hypothèse connue ethnographiquement, et relatée dans les textes historiques : celle de «pièges de chasse » (Desbrosse, 2011). Ces fosses fonctionneraient ainsi en batteries, à maillages plus ou moins lâches, dans des zones hors des habitats, près du passage des animaux sauvages. Les « trous » serviraient ainsi à piéger des animaux et les chasseurs pourraient avoir laissé certains « morceaux » de viande soit en offrandes, soit en rejets de découpe. Les rares animaux retrouvés complets dans ces fosses seraient ainsi des bêtes qui n'auraient pas été relevées à temps (Bontrond et al., 2013). Les hypothèses artisanales ou cultuelles ne sont toutefois pas définitivement réfutées (Achard-Corompt et al., 2010). Le modèle cynégétique reste par ailleurs à étayer par la découverte de vrais dispositifs de fosses à profil en $\mathrm{V}-\mathrm{Y}$, puisque la plupart de celles mises au jour sont retrouvées isolées.

Les trois exemples que nous présentons ici permettent une approche hypothético-déductive de l'interprétation cynégétique. Ils illustrent différents cas de figures qui entrent pleinement dans les problématiques de recherche sur ces fosses.

\section{DES FOSSES GROUPÉES : BÉTHENY « LE CHAMP DE MANEUVRE »}

À l'occasion de l'aménagement d'un parc d'activité entre Reims et Bétheny, un diagnostic archéologique préventif a été réalisé en 2011 au lieu-dit « le Champ de Manœuvre » (Garmond et Arnaud, 2011), sur un terrain de 18 ha. Ce diagnostic a permis la mise au jour de dix fosses à profil en V-Y, dont neuf sont groupées dans un même secteur géographique (fig. 2). Il est probable que la batterie comprenne un nombre encore plus grand de fosses.

Ce site est localisé sur un terrain aujourd'hui plan (l'altitude variant entre 84 et $85 \mathrm{~m} \mathrm{NGF).} \mathrm{Le} \mathrm{cours} \mathrm{d'eau}$ le plus proche est à plus de $2,5 \mathrm{~km}$. Le sous-sol est constitué de craie campanienne, recouverte par un horizon limoneux récent d'épaisseur variable.

Les dix fosses retrouvées présentent toutes les mêmes caractéristiques en surface : il s'agit de fosses ovales, apparaissant en surface de la craie, comblées par un limon brun granuleux. Les dimensions des fosses sont variables, deux types peuvent être distingués : un groupe de « grandes fosses » (en moyenne, $3 \times 1,7 \mathrm{~m})$ et un groupe de « petites fosses » $(2 \times 1,2 \mathrm{~m})$. Au stade du diagnostic, il 


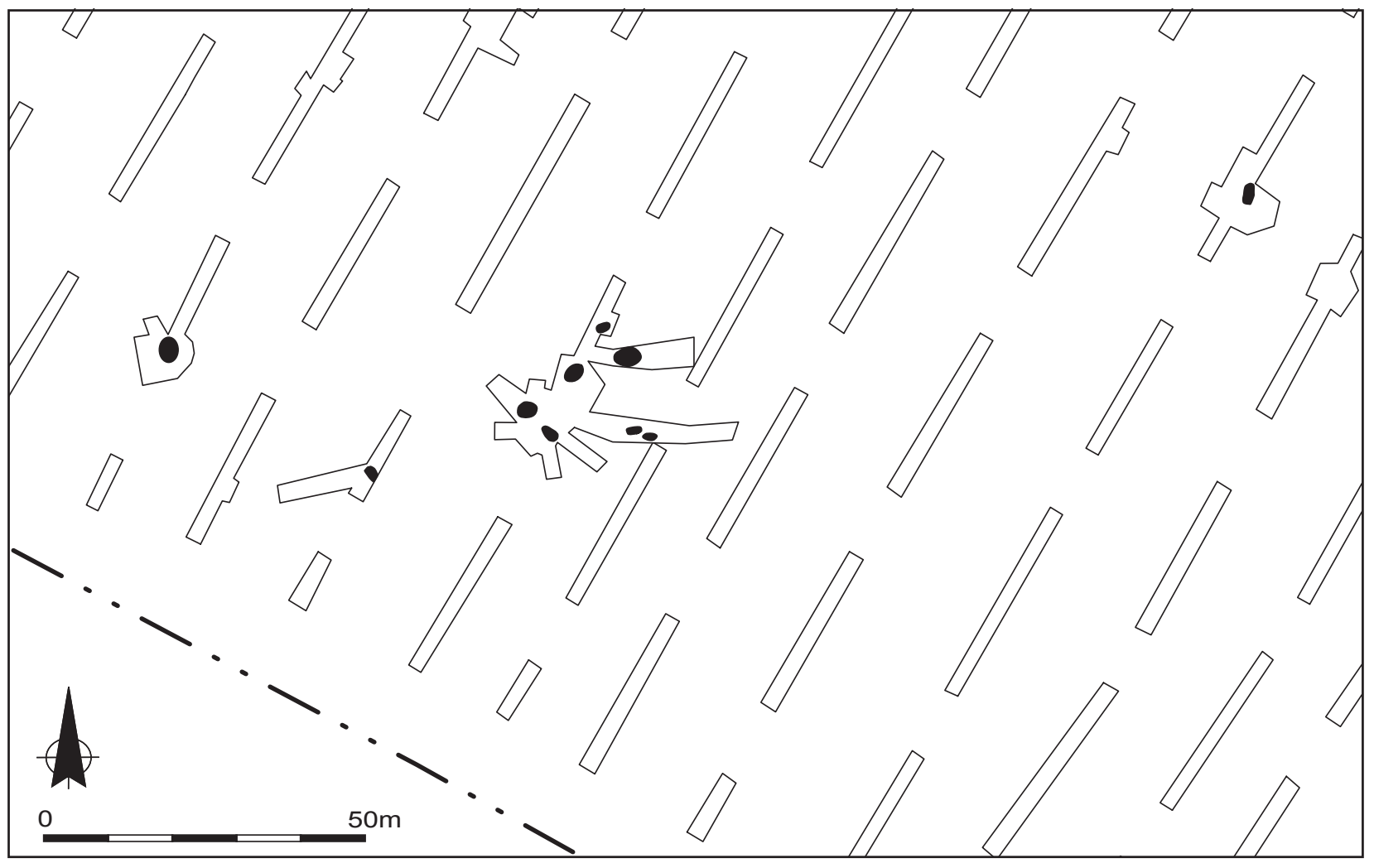

Fig. 2 - Plan de la batterie de fosses à profil en V-Y de Bétheny « le Champ de Manœuvre » (topographie F. Laudrin; DAO N. Garmond).

Fig. 2 - Plan of the slot-pits group, Bétheny 'Le Champ de Manœuvre'(topography F. Laudrin, CAD N. Garmond).

est impossible de préciser si ces groupes morphologiques ont une signification fonctionnelle ou chronologique.

L'orientation générale du groupe de fosses tend à être sud-ouest - nord-est. L'orientation de chaque fosse au sein de ce dispositif est cependant très variable, elle peut être sud-ouest - nord-est, sud-nord ou ouest-est. Aucune logique compréhensible à ce stade ne semble structurer les fosses entre elles.

Deux fosses ont fait l'objet d'un sondage mécanique par moitié dans le cadre du diagnostic (fig. 3). Elles montrent toutes deux un profil transversal typique en $\mathrm{Y}$, très étroit au fond. Les profondeurs sont de 1,7 et 2,45 m par rapport à la surface du substrat. Le comblement supérieur est constitué d'un limon brun homogène, tandis que la partie inférieure est plus crayeuse, alternant avec des passes limoneuses fines (fig. 4).

Ces fosses livrent, au diagnostic, très peu de mobilier. Une première livre en surface deux tessons attribuables à l'âge du Bronze ou au Hallstatt; une seconde livre, en surface également, deux tessons laténiens. Cependant, le mobilier issu du comblement supérieur des fosses à profil en $\mathrm{V}$-Y peut être postérieur à la période de fonctionnement de la fosse (Achard-Corompt et al., 2013). L'existence d'une ferme laténienne au nord (N. Garmond, en cours) explique probablement la présence de mobilier $d u$ second âge du Fer en surface d'une des fosses. Les prélèvements réalisés dans les deux fosses testées ont permis de recueillir une série de coquilles d'escargots. Celles-ci ont principalement été retrouvées dans le comblement supérieur, alors que dans le fond elles sont quasiment absentes. Une analyse malacologique sur des fosses similaires, retrouvées au sud de Reims à Bezannes (Bontrond et al., 2013), avait permis de conclure que le comblement supérieur des fosses était composé de niveaux de sols humifères « piégés » dans la fosse, tandis que le comblement du fond était plus rapide, probablement par effondrement du substrat crayeux.

Aucun élément ne permet donc, au diagnostic, de dater ces fosses, si ce n'est un terminus post quem à la Protohistoire. Hormis la ferme laténienne, aucun site néolithique ou protohistorique n'est attesté dans les environs, y compris sur le diagnostic de 18 ha. On peut raisonnablement attribuer ces fosses, par comparaison avec les fosses similaires bien datées en Champagne-Ardenne (cf. supra), au Néolithique ou à la Protohistoire ancienne, et supposer qu'elles sont éloignées de tout habitat contemporain.

Aucun élément direct ne permet non plus de conclure à leur fonction. La fouille de ces fosses s'avèrera donc essentielle pour permettre de préciser ces questions : le diagnostic seul ne permet de répondre aux problématiques posées par ce type de site.

En conclusion, le site du "Champ de Manœuvre " livre une batterie d'une dizaine de fosses à profil en $\mathrm{Y}$ et en comporte probablement plus. Ces fosses sont groupées sur un axe général SO-NE et semblent déconnectées de tout habitat contemporain. Elles possèdent donc 


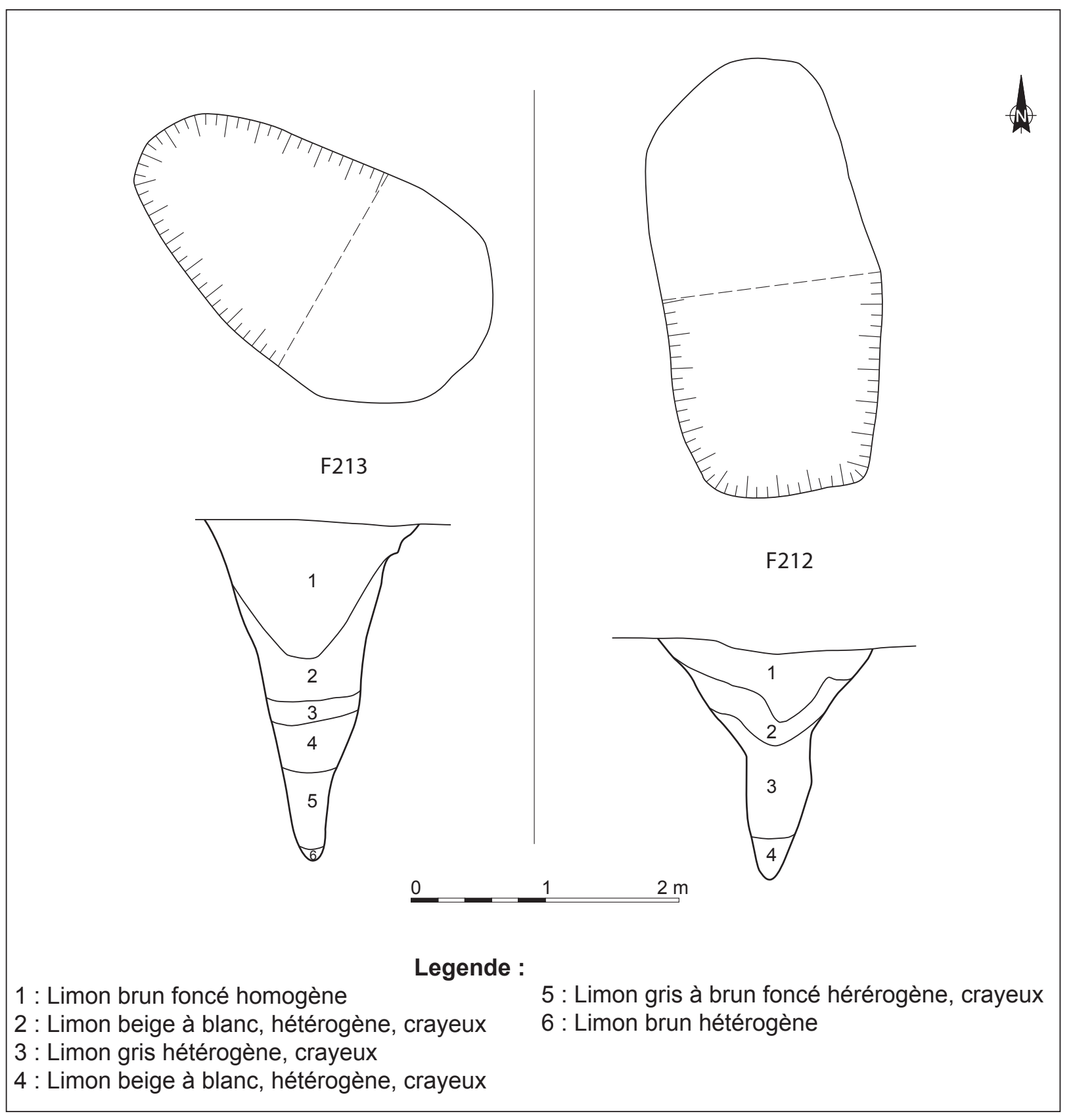

Fig. 3 - Plan et coupes des fosses à profil en V-Y de Bétheny « le Champ de Manœuvre » (DAO B. Panouillot).

Fig. 3 - Slot pit plans and sections, Bétheny 'Le Champ de Manœuvre'(CAD B. Panouillot).

des caractéristiques compatibles avec une interprétation cynégétique : il pourrait bien s'agir de pièges de chasse, hypothèse qui reste à conforter par de plus amples analyses.

\section{UN DISPOSITIF DE GRANDE AMPLEUR : BÉTHENY « LES ÉCAVÉS »}

\footnotetext{
A $1 \mathrm{~km}$ au nord, sur la même commune, à l'occasion de l'aménagement d'une zone d'activité au lieu-dit « les Écavés », deux diagnostics archéologiques préven-
}

tifs ont été réalisés en 2011 sur un total de 28 ha. Ces diagnostics ont permis la mise au jour d'un autre dispositif de fosses à profil en V-Y (Garmond et al., 2011 et Garmond, Huart et al., 2012).

Ces sondages ont été réalisés sur un terrain qui présente une pente nord-sud assez marquée, de 96 à 86 m NGF. Le cours d'eau le plus proche est localisé à plus de $3 \mathrm{~km}$. Le sous-sol est, de nouveau, constitué de craie campanienne, recouverte par un horizon limoneux peu épais.

Le dispositif mis au jour est constitué d'au moins dix fosses et en comporte probablement plus. Les fosses sont assez éloignées les unes des autres, la distance est d'au moins $50 \mathrm{~m}$ entre chaque fosse. Elles se répartissent sur 


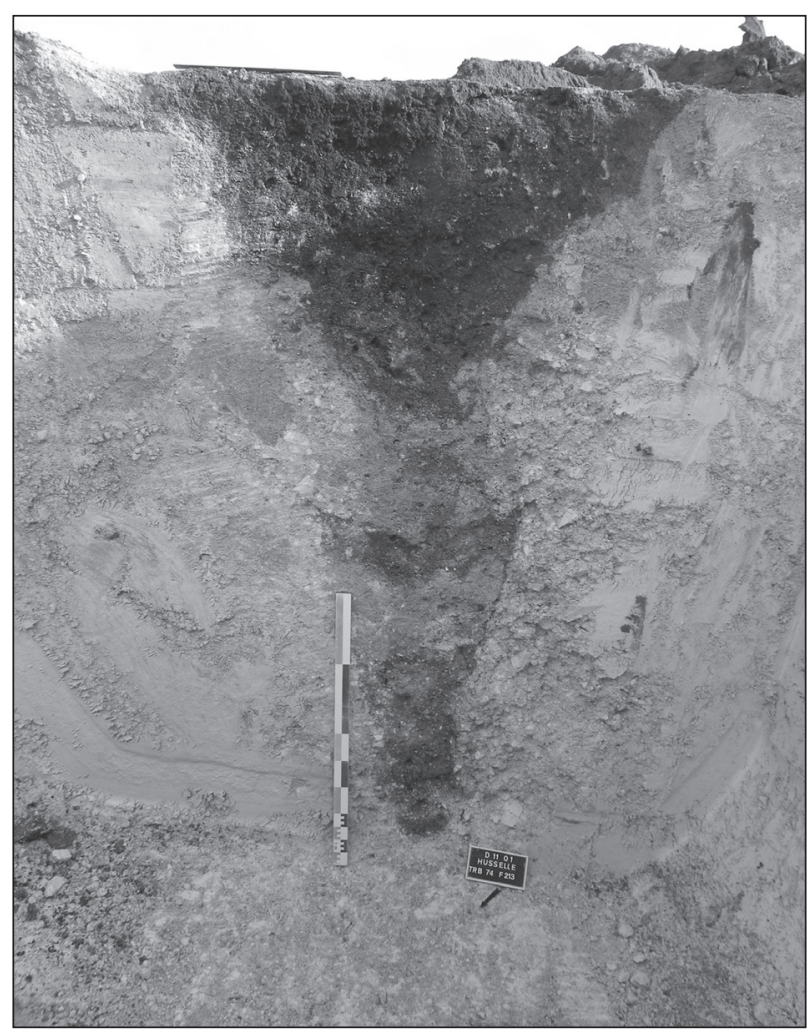

Fig. 4 - Coupe de la fosse 213 du « Champ de Manœuvre » (cliché P. Pautrat).

Fig. 4 - Section of slot pit 213, Bétheny 'Le Champ de Manæuvre' (photo P. Pautrat). un axe ouest-est qui a pu être suivi sur $500 \mathrm{~m}$ de long, et se poursuit probablement hors emprise dans les deux directions (fig. 5).

Les fosses, qui apparaissent en surface de la craie, présentent de nouveau des caractéristiques communes en surface (fig. 6), à savoir de grandes dimensions, la forme ovale et le comblement supérieur de limon brun granuleux. Les dimensions en surface sont assez variables : la plus petite fait $2,2 \times 1 \mathrm{~m}$, la plus grande $4,3 \times 2,1 \mathrm{~m}$. De nouveau, deux groupes de fosses semblent se distinguer, selon leurs dimensions, tant en surface qu'en profondeur. L'orientation des fosses est assez variable, sud-ouest nord-est, sud-nord ou nord-ouest - sud-est.

Cinq fosses ont été sondées mécaniquement dans le cadre des deux diagnostics, et une partie du dispositif fait l'objet d'une prescription de fouille. Les profils transversaux des fosses sondées sont en $\mathrm{V}$ ou en $\mathrm{Y}$, plus ou moins étroits (fig. 7). On peut constater que les fosses massives ont tendance à être moins étroites que les fosses les plus petites en surface. Les profondeurs, importantes, varient entre 1,8 et 2,38 $\mathrm{m}$ depuis la surface du substrat crayeux. On remarque que les fosses étroites ne sont pas nécessairement les moins profondes.

Les comblements peuvent être divisés en deux catégories. La moitié supérieure des fosses livre un limon brun et homogène, la moitié inférieure comporte des passes crayeuses litées avec de fines passes de limon brun. Parfois, mais non systématiquement, le fond est couvert d'une épaisseur d'une dizaine de centimètres de limon brun (fig. 8).

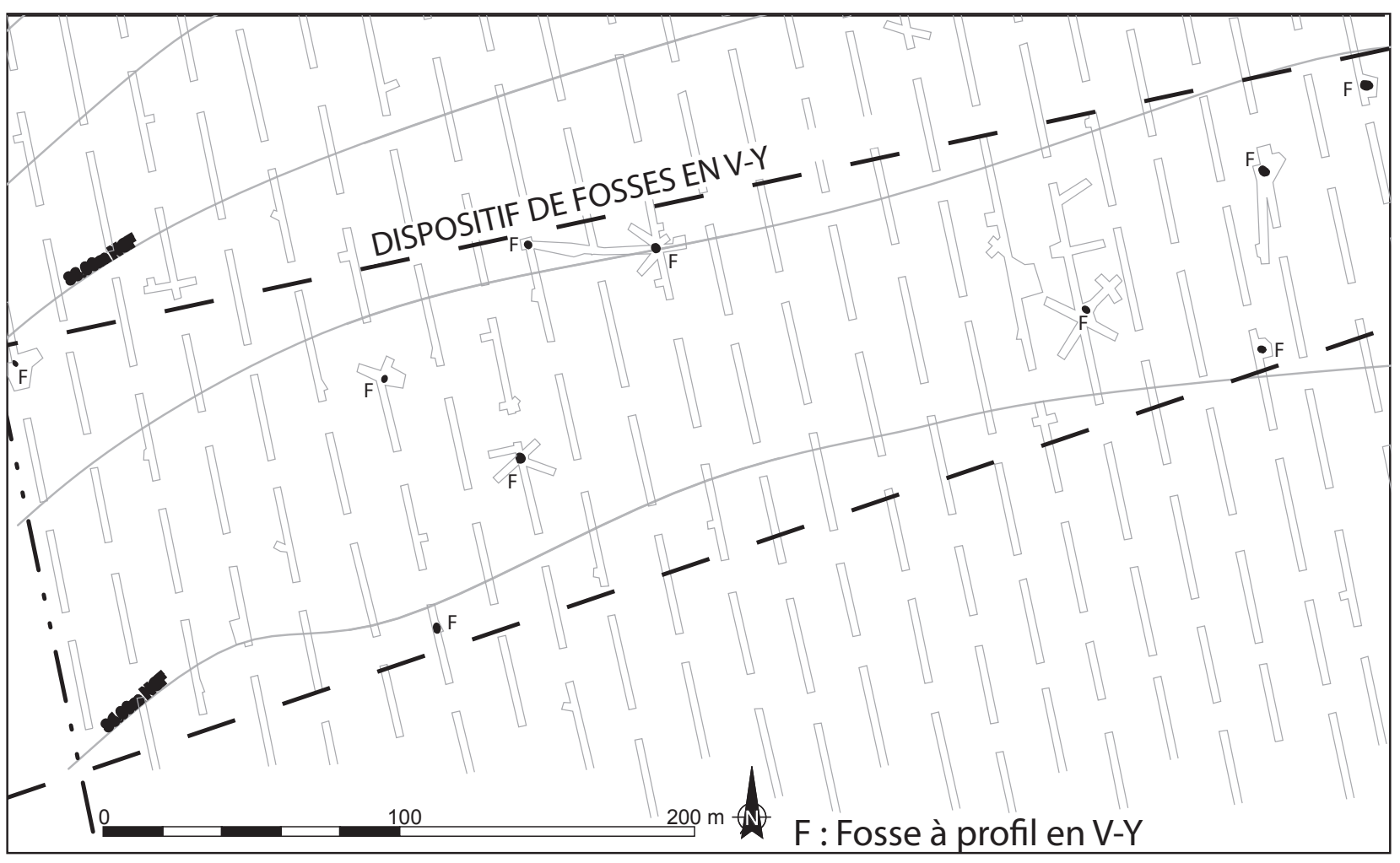

Fig. 5 - Plan du dispositif de fosses à profil en V-Y de Bétheny « les Écavés » (topographie F. Laudrin; DAO N. Garmond). Fig. 5 - Plan of the slot pits line, Bétheny 'Les Écavés'(topography F. Laudrin, CAD N. Garmond). 


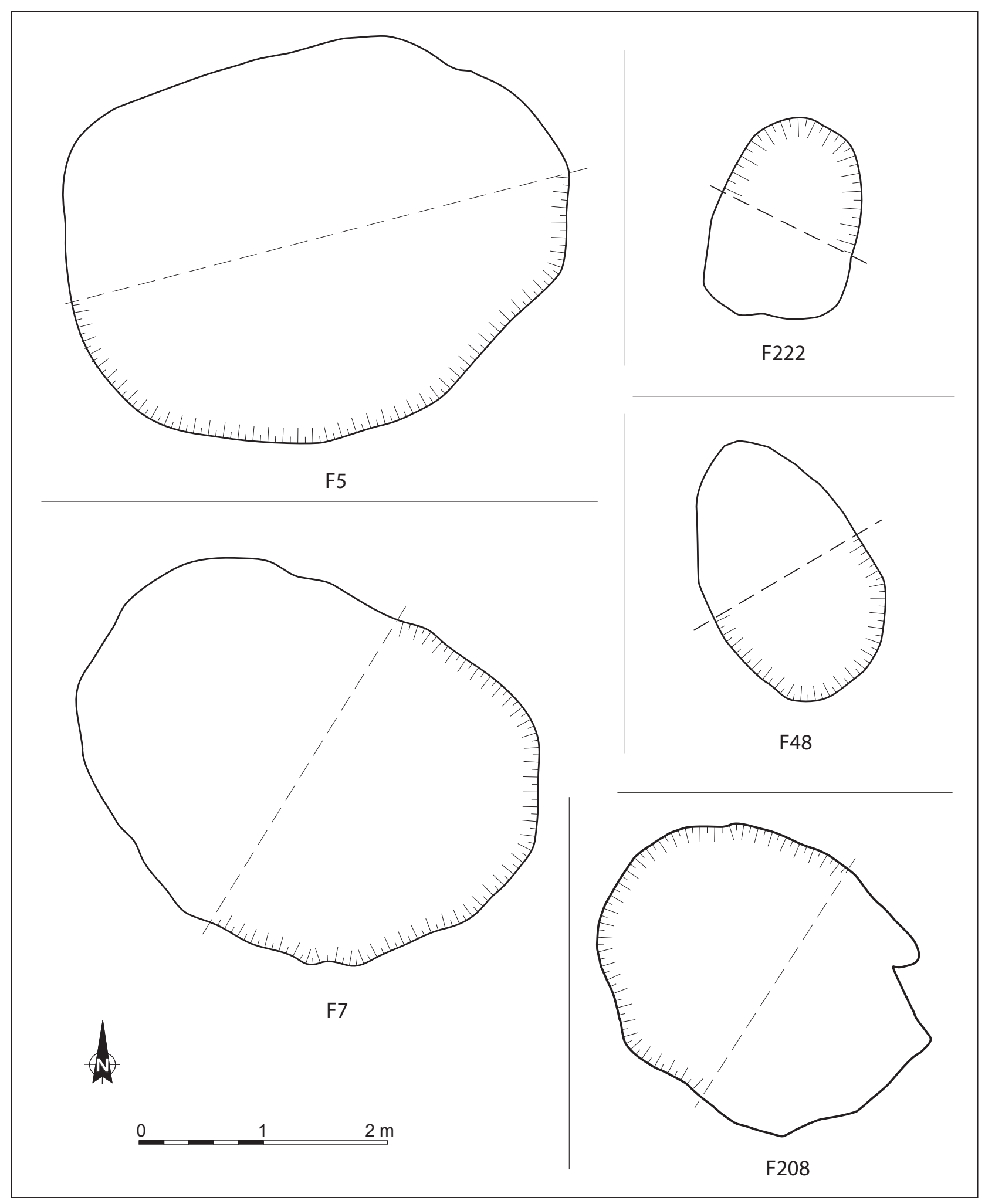

Fig. 6 - Plan des fosses à profil en V-Y de Bétheny « les Écavés » (DAO J. Hauret et S. Binder).

Fig. 6 - Plans of slot pits, Bétheny 'Les Écavés'(CAD J. Hauret and S. Binder). 


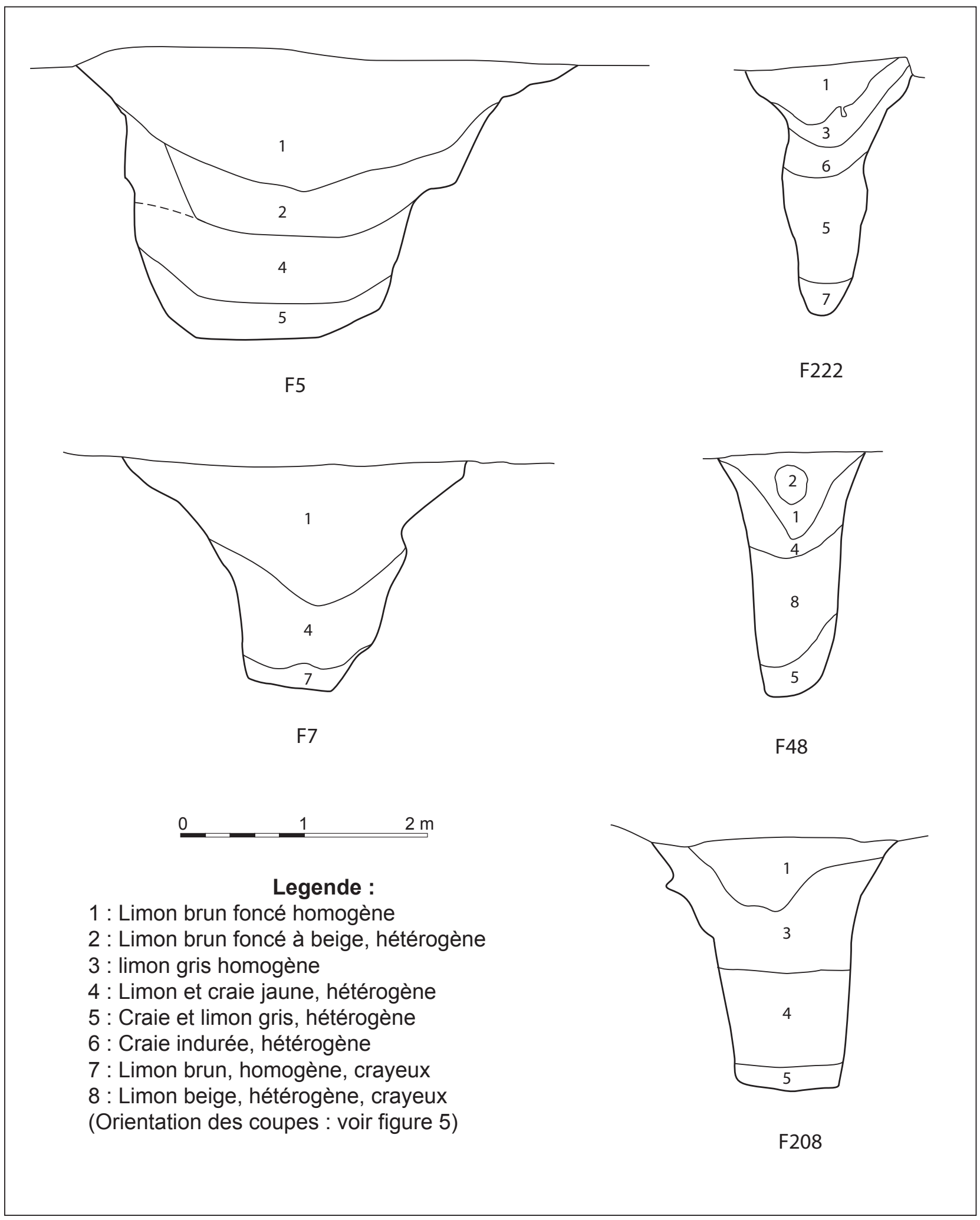

Fig. 7 - Coupe des fosses à profil en V-Y de Bétheny « les Écavés » (DAO J. Hauret et S. Binder).

Fig. 7 - Sections of slot pits, Bétheny 'Les Écavés'(CAD J. Hauret and S. Binder). 


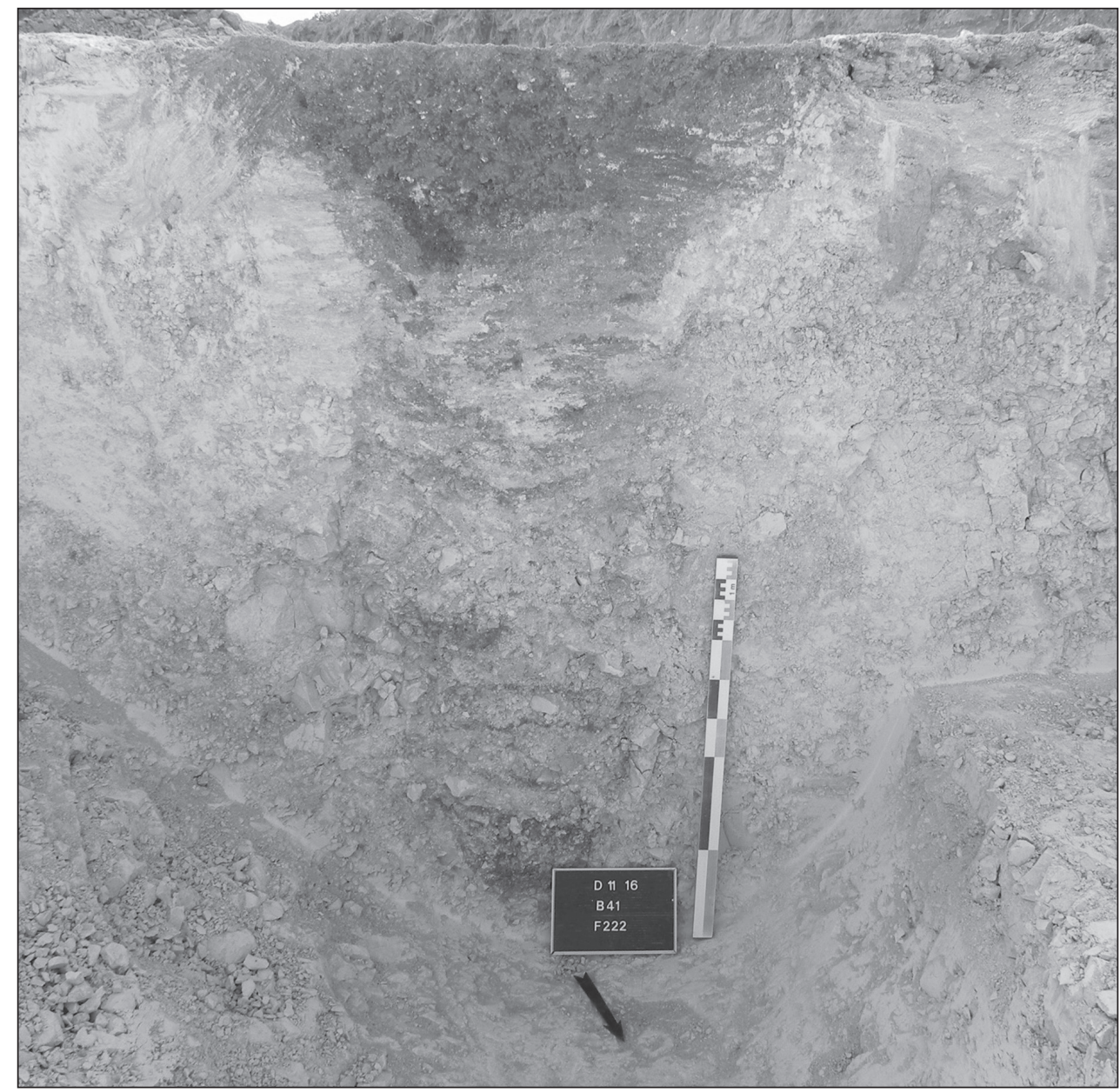

Fig. 8 - Coupe de la fosse 222 des « Écavés » (cliché I. Fournier).

Fig. 8 - Section of slot pit 222, 'Les Écavés' (photo I. Fournier).

Malgré des prélèvements systématiques qui ont été tamisés, aucun mobilier ne permet de dater ces fosses. Trois fosses présentent une nette concentration de coquilles d'escargots dans leur partie supérieure. Si l'on compare ce résultat avec d'autres fosses où des analyses malacologiques ont été entreprises (Bontrond et al., 2013), on peut en déduire que le remplissage sommital de ces fosses provient de sols humifères, autrement dit des niveaux de surface. À l'inverse, les parties inférieures crayeuses pourraient avoir été comblées plus rapidement. Le sommet de deux fosses livre d'ailleurs un très rare mobilier antique (tessons, clou) qui tendrait à indiquer un comblement simultané des fosses, probablement en lien avec une mise en culture des terres lors de l'implantation à proximité d'un établissement rural durant l'Antiquité.

$\mathrm{Si}$ ces fosses sont toutes contemporaines, ce qui reste à démontrer, elles seraient attribuables au Néolithique ou à la Protohistoire (cf. supra). Or, de nouveau, aucun habitat de ces périodes n'est attesté sur les 28 ha de diagnostic ou dans les environs proches. Seul un silo du Hallstatt, retrouvé plus au nord, pourrait éventuellement être associé, mais sa contemporanéité avec les fosses est loin d'être assurée (Garmond et al., 2011).
L'organisation spatiale des fosses semble parfaitement compatible avec l'hypothèse d'un dispositif cynégétique. En effet, les dix fosses sont toutes situées sur une même courbe de niveau (entre 86 et 88 m NGF), sur une pente nord-sud assez marquée. En d'autres termes, ces fosses viennent barrer la pente naturelle du terrain, tout en étant suffisamment espacées pour assurer des points de passages larges. Si le dispositif a pu être suivi sur $500 \mathrm{~m}$ de longueur, il s'étend encore probablement à l'ouest et à l'est. On peut ainsi très bien imaginer un dispositif de chasse, à maillage lâche, barrant une pente naturelle, peut-être à un point de passage d'animaux sauvages. La taille des fosses, ainsi que leur profil, pourrait dépendre du type d'animal chassé. Un exemple, similaire par son envergure, est connu en Allemagne, dans le BadeWurtemberg, où trois lignes de Schlitzgruben sont disposées en demi-cercle, sur une étendue de 0,5 ha (Friederich, 2013).

Demeurant au stade du diagnostic, cette hypothèse, aussi tentante soit-elle, reste à conforter. De nouveau, on peut espérer qu'une fouille vienne répondre aux questions liées à la chronologie et au fonctionnement de ces fosses. Néanmoins, ce dispositif reste exceptionnel par son étendue. 


\section{UNE BATTERIE DANS UN VILLAGE DU RUBANÉ : SAINT-MARTIN-SUR-LE-PRÉ « RUE DES CASTORS »}

\section{Le site}

À l'occasion de la construction de logement rue des Castors à Saint-Martin-sur-le-Pré, dans la périphérie ouest de Châlons-en-Champagne, un diagnostic archéologique en 2007 puis deux fouilles, en 2010 et en 2011, ont été réalisés sur un terrain de $5400 \mathrm{~m}^{2}$ (Garmond, dir., 2012). La fouille a permis de révéler, parmi d'autres occupations archéologiques, une partie d'un village du Rubané, comprenant une batterie de fosses à profil en V-Y (fig. 9).

Le site est localisé sur la terrasse alluviale ancienne de la Marne, en surplomb de la rivière actuelle qui coule en contrebas. Le terrain présente une légère pente nord-est sud-ouest (entre 85 et $84 \mathrm{~m} \mathrm{NGF).} \mathrm{Le} \mathrm{sous-sol} \mathrm{est} \mathrm{très}$ variable, constitué de limons plus ou moins crayeux dans lesquels s'ouvrent les structures, recouvrant des horizons de grèves alluviales anciennes.

Le village du Néolithique ancien est composé d'au moins trois maisons, probablement plus, dont une maison longue, associées à leurs fosses latérales et à trois sépultures (Garmond, Binder et al., 2012). Le village, s'étendant hors emprise, suit un plan assez classique pour la période, avec des maisons orientées nord-ouest - sudest. Le mobilier, retrouvé notamment dans les fosses de construction, est abondant et comprend de la céramique, de l'industrie lithique, de la faune, du macro-outillage, du torchis et de la parure en coquillage issue des tombes. Ce mobilier est attribuable dans son intégralité au Rubané récent du Bassin parisien (RRBP). Le site a été occupé entre 5200 et 4800 avant J.-C., probablement même entre 5100 et 4900 avant J.-C. en fourchette restreinte.

\section{La batterie de fosses à profil en V-Y}

Au sein même de ce village, une batterie de sept fosses à profil en V-Y a été mise au jour (fig. 9).

Ces fosses sont assez similaires : en surface, elles sont ovales, avec des longueurs variant entre 2,3 et $3,5 \mathrm{~m}$ et des largeurs entre 0,64 et 1,8 m (fig. 10). L'orientation des fosses est très variable. Les profils transversaux sont en $\mathrm{V}$ ou $\mathrm{Y}$, avec des profondeurs allant de 0,86 à $1,8 \mathrm{~m}$ depuis la surface du décapage (fig. 11). Les profils longitudinaux sont assez asymétriques et ne présentent aucun aménagement particulier permettant de mieux comprendre la fonction des fosses. La fosse 48 , située à proximité d'une maison danubienne, pourrait avoir été réaménagée dans sa moitié supérieure pour servir de fosse-dépotoir, comme en atteste le mobilier présent (cf. infra). La fosse 2 est moins profonde que les autres, mais l'arasement important de la partie nord du site pourrait en être la cause, aussi peut-on la considérer comme initialement similaire aux autres fosses du même type.

Les comblements des fosses sont multiples, stratifiés (fig. 12). Ils peuvent cependant être groupés en deux ou trois familles selon les fosses : le comblement supérieur est plutôt limoneux, brun, homogène; le comblement inférieur alterne passes crayeuses et passes limoneuses. Souvent, un comblement de limon brun marque le fond des fosses.

Cinq des sept fosses sont très proches et sont organisées sur un arc de cercle orienté ouest-est (fig. 9), qui s'interrompt à l'ouest sur l'avant d'une maison danubienne arasée : la maison semble constituer une limite « physique » à la batterie de fosse. La limite à l'est, hors emprise, n'est pas connue.

\section{Le mobilier des fosses de la batterie}

Le mobilier retrouvé dans les fosses à profil en $\mathrm{V}$-Y, bien que réduit à échelle du site, est abondant pour ce type de structures. En effet, alors qu'en Champagne-Ardenne seul $14,6 \%$ des fosses à profil en V-Y livrent du mobilier (Achard-Corompt et al., 2010), à Saint-Martin-sur- le-Pré, cinq des sept fosses comportent du matériel archéologique (soit 71,4\%). Dans le comblement supérieur de cinq fosses, on retrouve en effet des fragments de céramiques néolithiques, de l'industrie lithique, de la faune et quelques graines. Cependant, l'élément le plus remarquable est la présence, à $-1,2 \mathrm{~m}$ dans la fosse 19 , d'une portion de cervidé retrouvée partiellement en connexion anatomique.

\section{La céramique}

Du mobilier céramique a été récolté dans les couches sommitales de cinq fosses en V-Y. La fosse 48 livre le lot céramique le plus important (32 fragments), d'un poids total de $331 \mathrm{~g}$. L'état de grande fragmentation du mobilier est notable, comme pour les fosses $15,23,19$ et 32 , qui livrent seulement cinq tessons.

La fosse 48 a livré un individu-vase de céramique épaisse (fig. 13, $\mathrm{n}^{\circ}$ 1). Par sa forme, son décor digité qui s'inscrit dans un système décoratif en guirlande, et son dégraissant coquillé très abondant, ce vase possède des caractéristiques similaires à ceux du corpus céramique du Néolithique ancien du site. Deux autres tessons, issus de la même fosse, possèdent des décors réalisés en impressions au peigne. L'épaisseur de leurs parois oscille entre 0,4 et $0,6 \mathrm{~mm}$. Le premier arbore un décor au peigne à deux dents, en impression séparée, pouvant s'inscrire dans un décor de type chevron (fig. 13, n 2). Le second laisse figurer des incisions parallèles, tracées vraisemblablement à l'aide d'un peigne (fig. 13, no 3 ). Un autre tesson comporte un décor réalisé avec un outil de section circulaire, type bâtonnet (fig. 13, no 4).

Un tesson de céramique fine, décoré, a également été retrouvé dans la fosse 19. La surface externe, très érodée, laisse distinguer les traces d'une impression au peigne, sans pouvoir déterminer le nombre de dents, ni le mode d'impression.

Les tessons retrouvés dans les fosses 15, 23 et 32 n'ont pas livré d'éléments typologiques ou stylistiques. L'observation des groupes de pâtes autorise néanmoins le rapprochement de ces tessons au corpus du Néolithique ancien. 


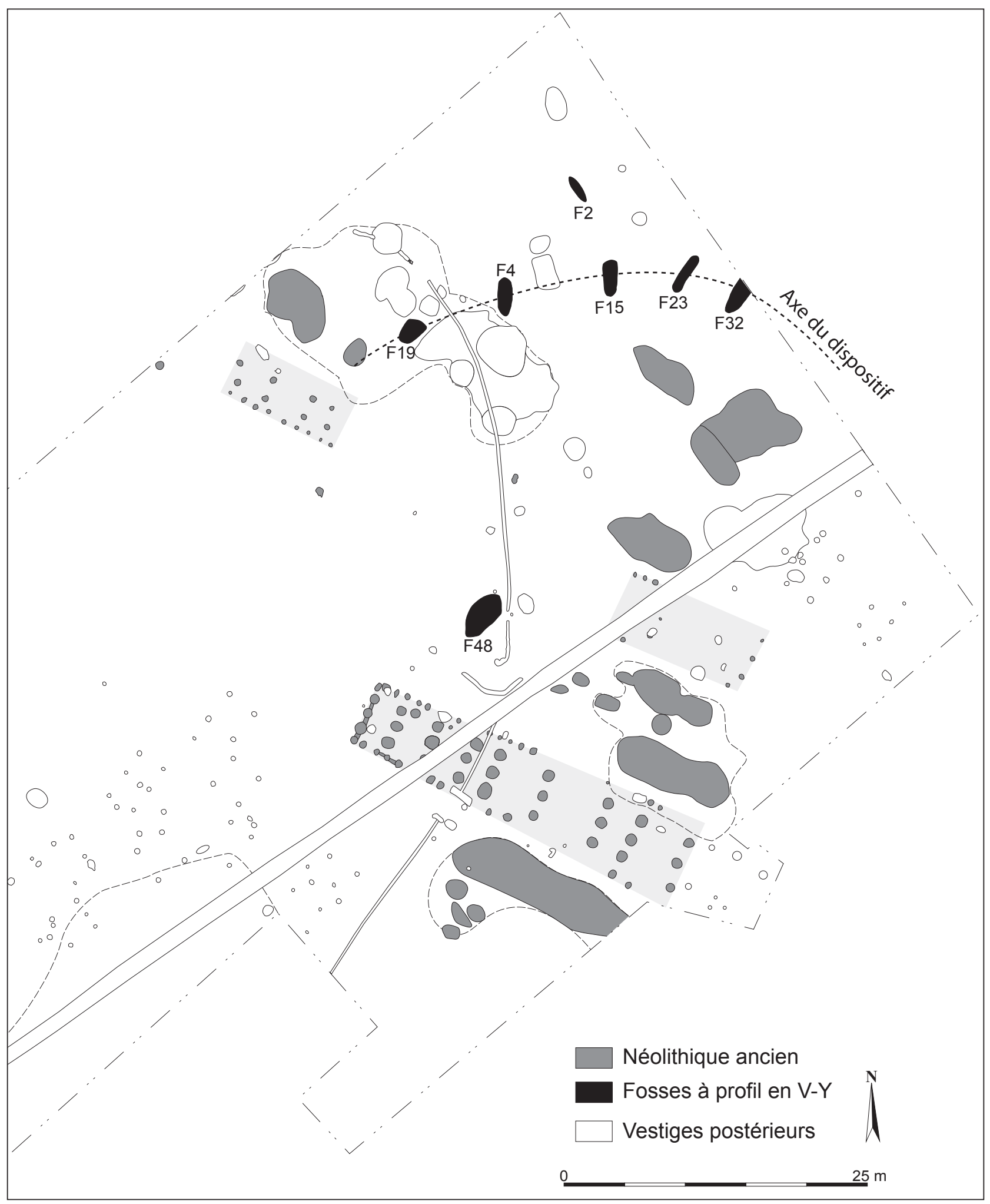

Fig. 9 - Plan de l'occupation du Néolithique ancien de la « rue des Castors » à Saint-Martin-sur-le-Pré (topographie F. Laudrin; DAO N. Garmond).

Fig. 9 - Plan of the early Neolithic site of Saint-Martin-sur-le-Pré 'Rue des Castors'(topography F. Laudrin, CAD N. Garmond). 


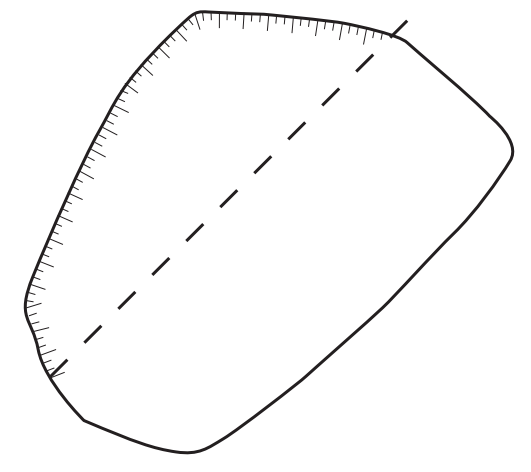

F19

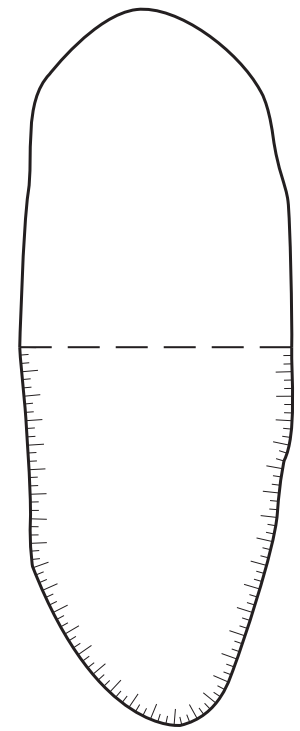

F4

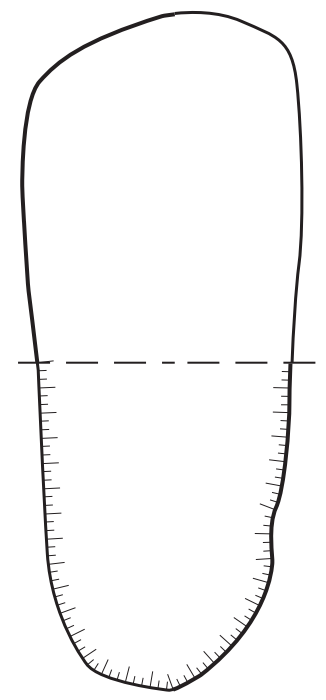

F15

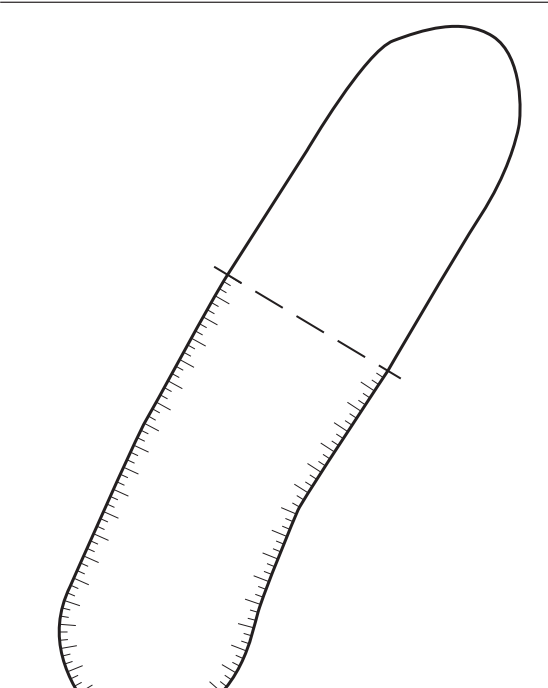

F23

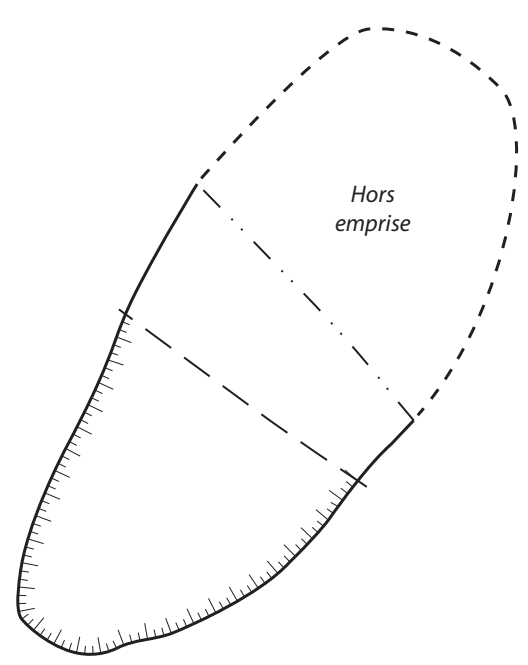

F32
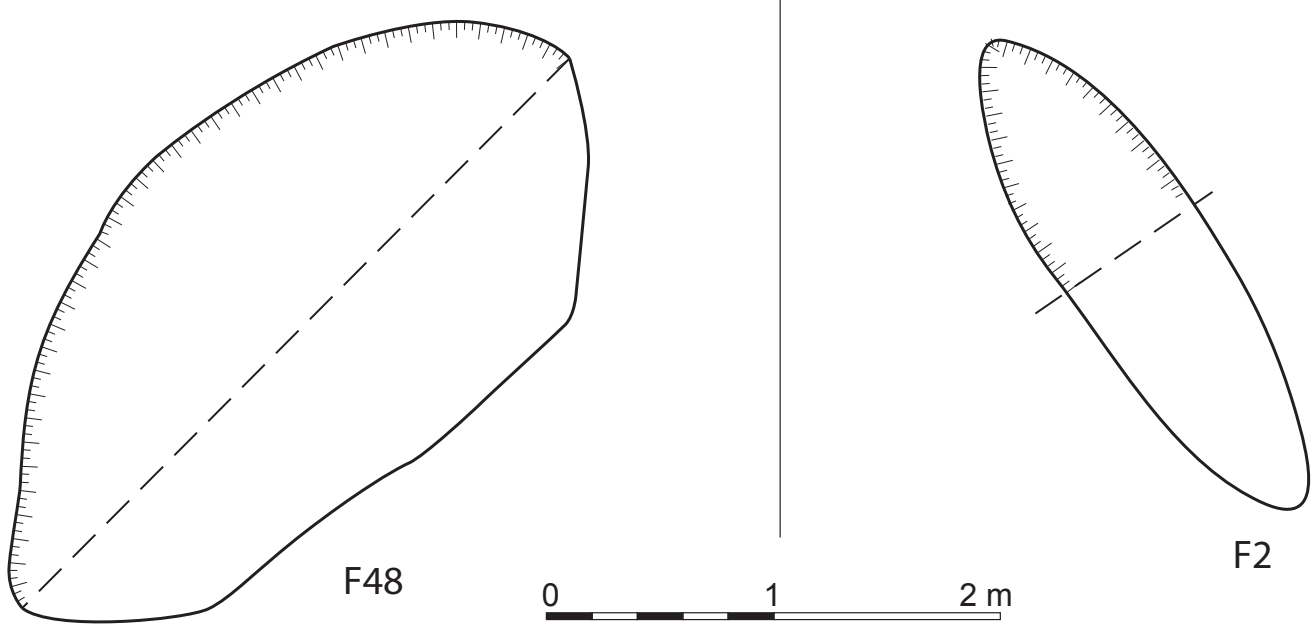

F2

m

Fig. 10 - Plan des fosses à profil en V-Y de la « rue des Castors » à Saint-Martin-sur-le-Pré (DAO D. Rennesson).

Fig. 10 - Plan of the slot pits, 'Rue des Castors' (CAD D. Rennesson). 


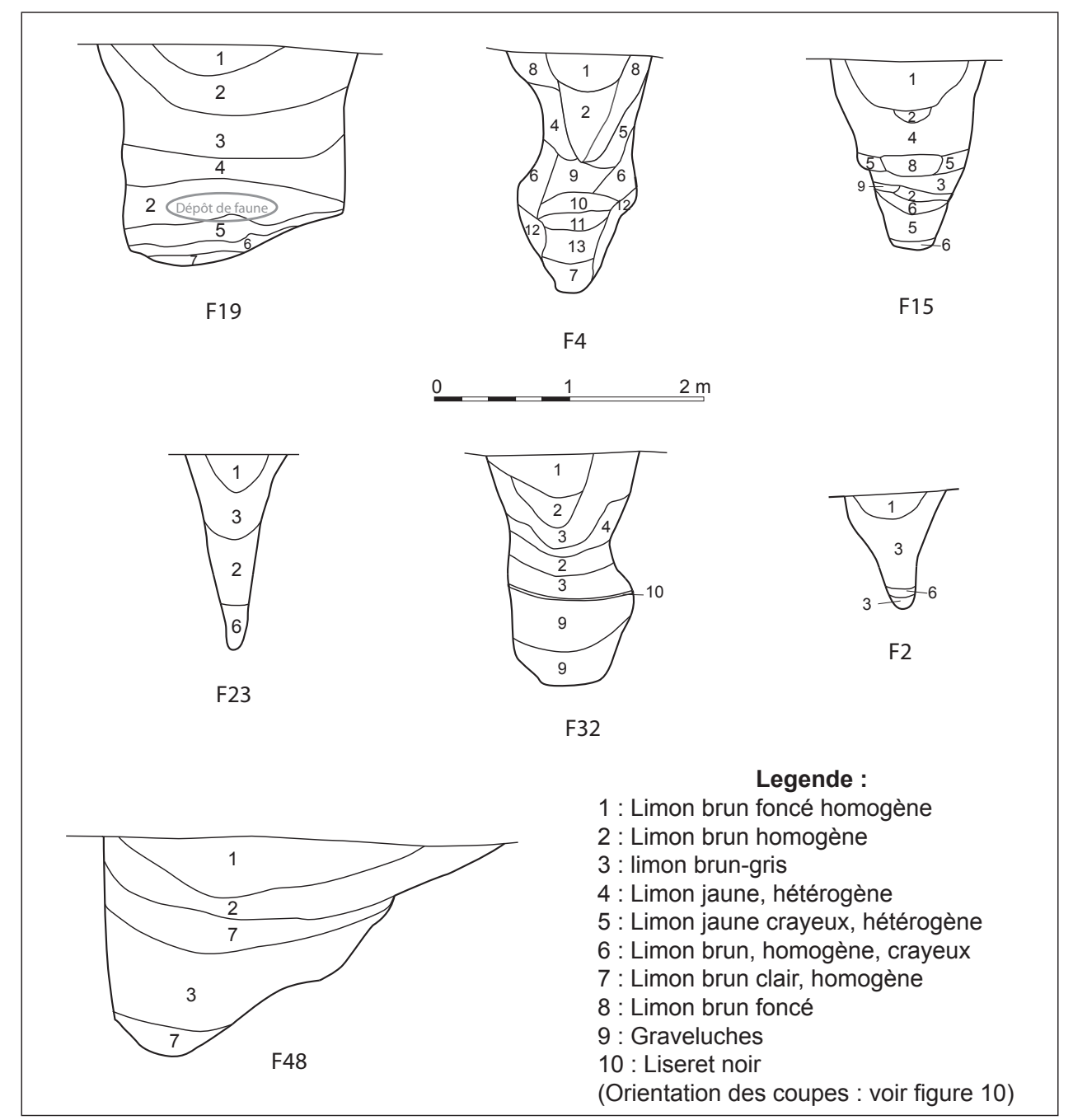

Fig. 11 - Coupes des fosses à profil en V-Y de la « rue des Castors » à Saint-Martin-sur-le-Pré (DAO D. Rennesson).

Fig. 11 - Sections of the slot pits, 'Rue des Castors' (CAD D. Rennesson).

Fig. 12 - Coupe de la fosse 23 de la « rue des Castors » (cliché D. Rennesson).

Fig. 12 - Section of slot pit 23, 'Rue des Castors' (photo D. Rennesson).

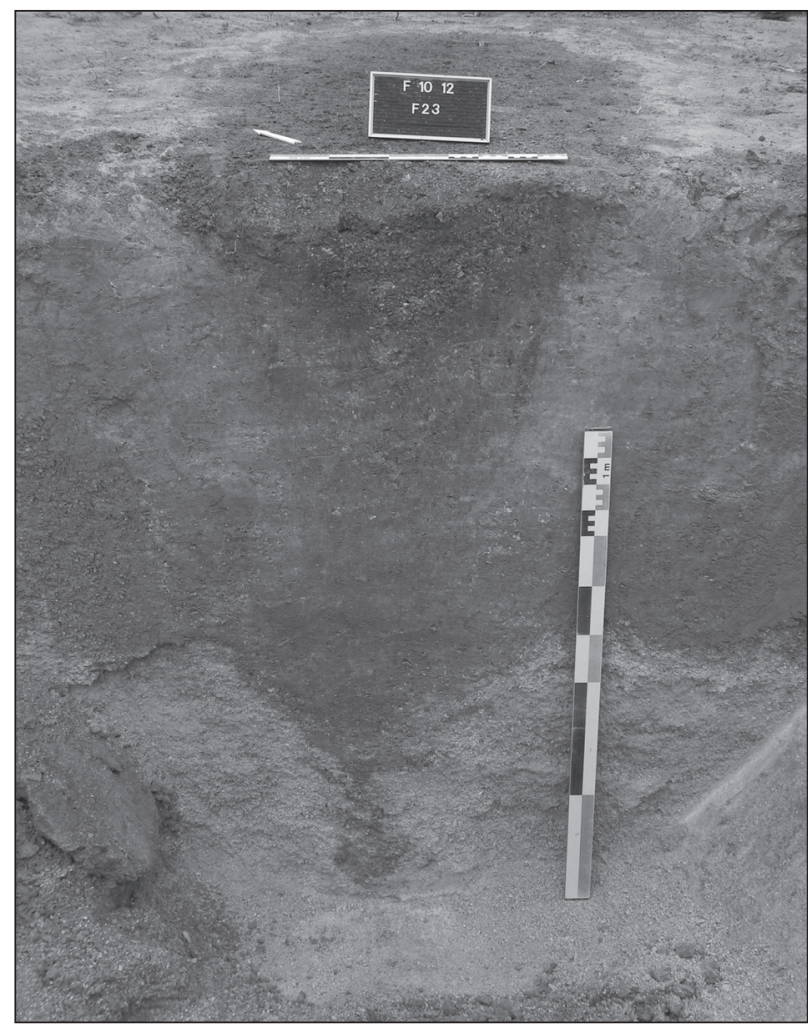




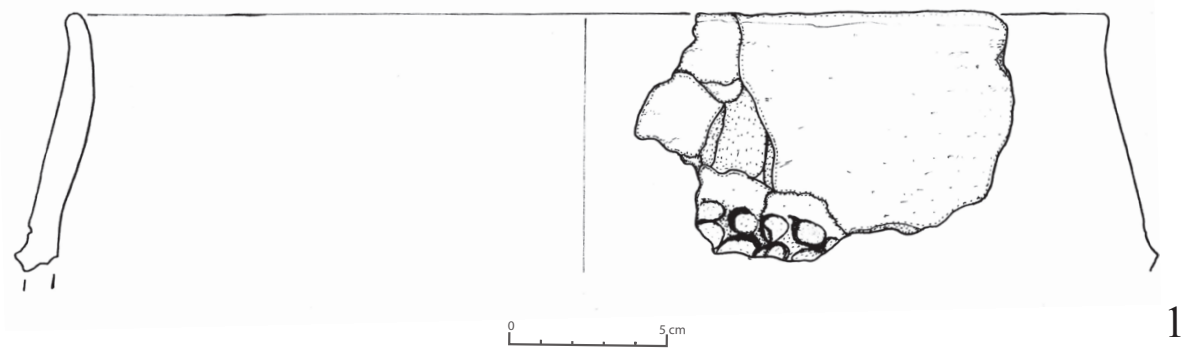

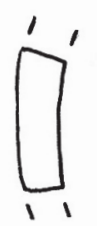

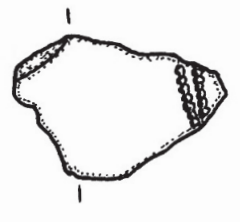

2

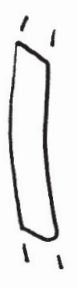

0

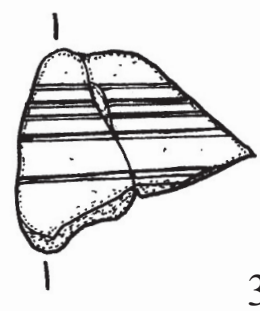

3
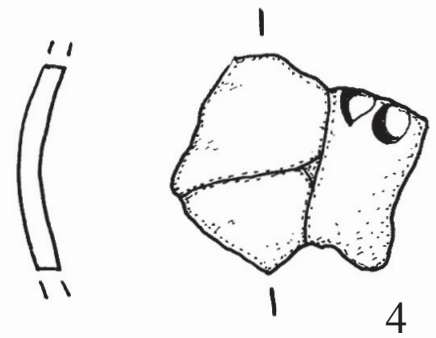

Fig. 13 - Céramiques de la fosse 48 de la « rue des Castors » (dessins S. Binder).

Fig. 13 - Pottery sherds from slot pit 48, 'Rue des Castors'(drawings S. Binder).

\begin{tabular}{|c|c|c|c|c|c|c|c|}
\hline & & \multicolumn{2}{|c|}{ Taxons domestiques } & \multicolumn{2}{|c|}{ Taxons sauvages } & \multirow{2}{*}{ Indéterminés } & \multirow{2}{*}{ Total } \\
\hline \multicolumn{2}{|c|}{ En nombre de restes } & Bœuf & Capriné & Aurochs & Cerf & & \\
\hline F15 & Niveau sup. & - & - & - & - & 1 & 1 \\
\hline \multirow{2}{*}{ F19 } & Niveau sup. & - & - & 2 & 1 & - & 3 \\
\hline & Fond & - & - & - & 1 & - & 1 \\
\hline F23 & Niveau sup. & 1 & - & - & - & 1 & 2 \\
\hline F48 & Niveau sup & 24 & 2 & - & 8 & 140 & 174 \\
\hline
\end{tabular}

\begin{tabular}{|l|l|c|c|c|c|c|c|}
\multicolumn{2}{|c|}{ En poids des restes $(\mathrm{g})$} & Bœuf & Capriné & Aurochs & Cerf & Indéterminés & Total \\
\hline F15 & Niveau sup. & - & - & - & - & 5 & 5 \\
\hline \multirow{2}{*}{ F19 } & Niveau sup. & - & - & 96 & 16 & - & 112 \\
\cline { 2 - 8 } & Fond & - & - & - & 69 & - & 69 \\
\hline F23 & Niveau sup. & 5 & - & - & - & 1 & 6 \\
\hline F48 & Niveau sup. & 1245 & 11 & - & 28 & 380 & 1664 \\
\hline
\end{tabular}

Tabl. 1 - Fréquence des taxons présents dans les fosses à profil en V-Y de la « rue des Castors » (exprimée en nombre et poids de restes). Table 1 - Species frequency in slot pits, 'Rue des Castors' (expressed as number and weight of identified bones). 
L'étude du matériel de ces fosses, mise en corrélation avec les résultats issus de la lecture des lames minces (réalisée par G. Fronteau), montre que ce matériel est identique à celui retrouvé dans les autres fosses du village Rubané du site. Les céramiques issues des fosses à profil en V-Y du site de Saint-Martin-sur-le-Pré trouvent donc leur place au sein d'un corpus abondant, riche en informations stylistiques et technologiques, se rattachant à l'étape moyenne du RRBP telle que définie dans la vallée de l'Aisne (Constantin et Ilett, 1997).

\section{La faune}

Parmi les sept fosses à profil en V-Y, quatre ont fourni des vestiges osseux (tabl. 1). Le nombre de restes est compris entre 1 et 174 ossements. À l'exception de la fosse 19 , qui a livré des restes osseux aussi bien au sommet qu'au fond de son comblement, la majorité de ces assemblages fauniques provient du comblement supérieur de ces fosses (F15, F23, F48).

Les os déterminés (F19, F23 et F48) sont attribuables à des espèces domestiques et/ou sauvages. Les restes de gibiers sont exclusifs au sein de la fosse 19, tandis que les restes de taxons domestiques dominent au sein de la fosse 48 .

Dans le comblement supérieur de la fosse 19, l'aurochs et le cerf sont figurés respectivement par un fragment de scapula et de côte, le cerf par une vertèbre lombaire. Les restes de cerf mis en évidence à $1,20 \mathrm{~m}$ de profondeur correspondent à un ensemble anatomique constitué de trois vertèbres thoraciques en connexion associées à deux plats de côtes de cerf (fig. 14), comptés comme un seul reste.

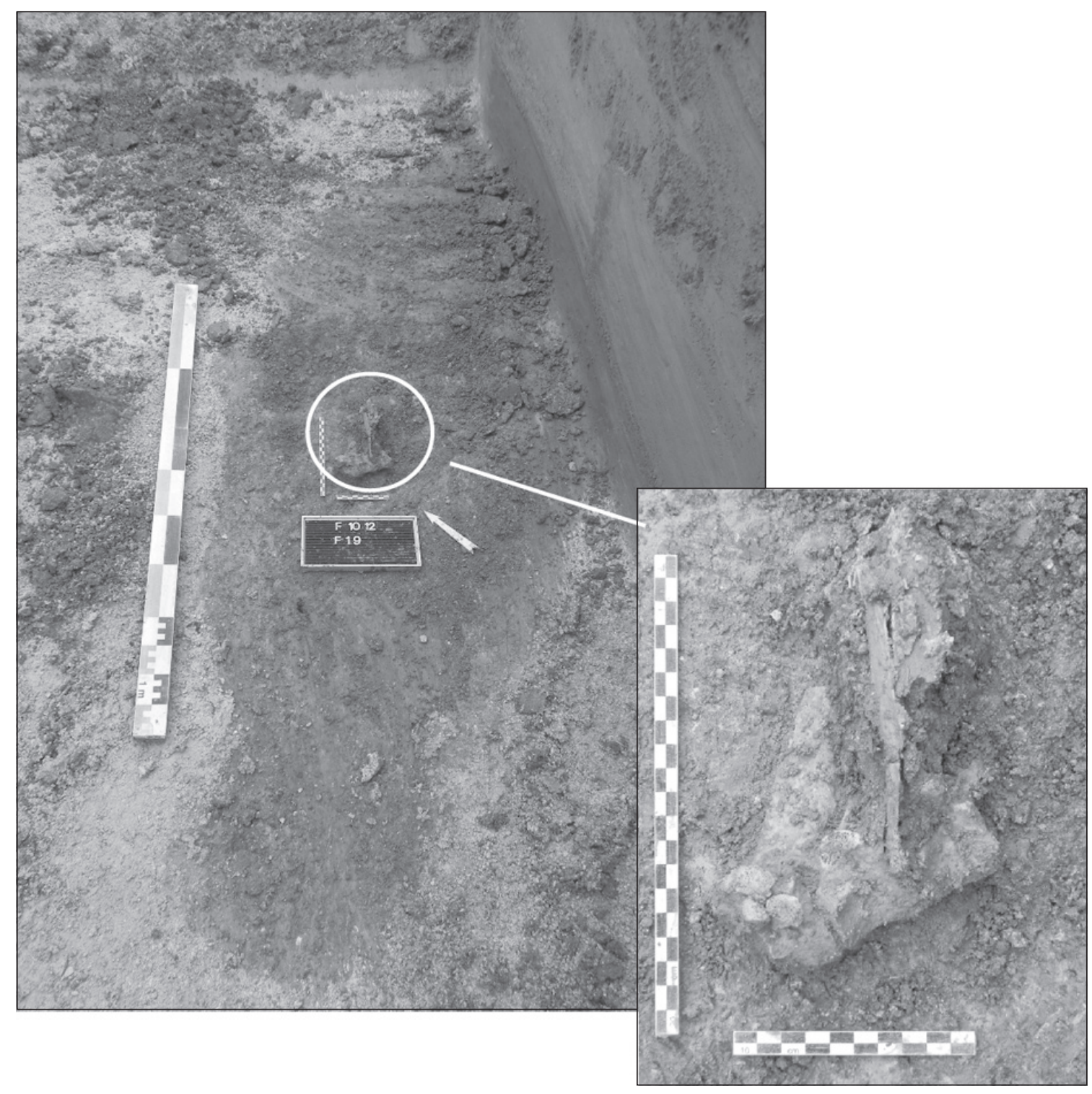

Fig. 14 - Localisation et détail des trois vertèbres thoraciques et des deux plats de côtes de cerf mis en évidence dans la fosse 19 de la «rue des Castors » (clichés F. Poupon).

Fig. 14 - Location and detail of three thoracic vertebrae and two ribs of deer excavated in slot pit 19, 'Rue des Castors' (photos F. Poupon). 
Au sein de la fosse 48, les restes de bœuf dominent. Ils proviennent des régions anatomiques du rachis, des membres antérieurs et postérieurs, ainsi que des bas de pattes (tabl. 2). L'importance du cerf (huit restes) est à relativiser en raison du nombre élevé de fragments de bois (sept fragments sur huit restes de cerf).

Aux restes osseux issus des niveaux supérieurs de comblement de ces fosses à profil en $\mathrm{V}$-Y sont également associés systématiquement des fragments de céramiques (cf. supra). Le caractère domestique de ces rejets est renforcé par la présence d'ossements brûlés et découpés d'animaux d'élevage au sein de la fosse 48. La rareté des restes osseux mis en évidence dans le comblement supérieur des fosses 15 et 23 leur confère un aspect résiduel, également lié à une activité domestique issue des unités d'habitation rubanées proches (présence d'une phalange de bœuf domestique et d'un fragment d'os carbonisé dans la fosse 23).
À l'inverse, l'ensemble anatomique de cerf mis en évidence dans le fond de la fosse 19 tranche avec le mobilier des comblements supérieurs. Dans le cas d'une fosse isolée, ces vertèbres thoraciques en connexion, associées à ces deux plats de côtes de cerf, auraient pu être interprétées comme les rejets issus d'une activité cynégétique, hypothèse jusqu'ici généralement privilégiée lors d'une telle découverte (Saurel et al., 2006; Friederich, 2013; Bontrond et al., 2013). Néanmoins, la proximité et surtout la contemporanéité de cet assemblage faunique avec l'habitat dénotent par rapport au caractère habituellement isolé, tant spatialement que chronologiquement, de ce type de fosses et de dépôts. S'agit-il toujours d'un piège à gibier encore en fonction juste avant l'installation de l'habitat rubané? Ou, sachant que la faune associée aux unités d'habitation est presque dépourvue de vertèbres et de côtes de cerf (parmi les 114 restes de cerf identifiés au sein des différentes unités d'habitation du rubanée,

\begin{tabular}{|c|c|c|c|c|c|}
\hline & & & NR & PDR (g) & NMPS \\
\hline \multirow{16}{*}{ Bœuf } & \multirow{3}{*}{ Rachis } & atlas & 2 & 53 & 1 \\
\hline & & cervicale & 1 & 18 & 1 \\
\hline & & thoracique & 2 & 42 & 1 \\
\hline & Total rachis & & 5 & 113 & 3 \\
\hline & \multirow{4}{*}{ Membres antérieurs } & scapula & 3 & 32 & 1 \\
\hline & & humérus & 1 & 217 & 1 \\
\hline & & radius & 5 & 558 & 4 \\
\hline & & ulna & 1 & 24 & 1 \\
\hline & Total membres antérieurs & & 10 & 831 & 7 \\
\hline & \multirow{3}{*}{ Membres postérieurs } & coxal & 1 & 46 & 1 \\
\hline & & fémur & 2 & 81 & 2 \\
\hline & & tibia & 4 & 70 & 2 \\
\hline & Total membres postérieurs & & 7 & 197 & 5 \\
\hline & \multirow{2}{*}{ Bas de patte } & mtc & 1 & 91 & 1 \\
\hline & & pha1 & 1 & 13 & 1 \\
\hline & Total bas de patte & & 2 & 104 & 2 \\
\hline Total bœuf & & & 24 & 1245 & 17 \\
\hline \multirow{4}{*}{ Capriné } & Membres postérieurs & fémur & 1 & 5 & 1 \\
\hline & Total membres postérieurs & & 1 & 5 & 1 \\
\hline & Bas de patte & $\mathrm{mtt}$ & 1 & 6 & 1 \\
\hline & Total bas de patte & & 1 & 6 & 1 \\
\hline Total capriné & & & 2 & 11 & 2 \\
\hline \multirow{4}{*}{ Cerf } & Tête & bois & 7 & 15 & 1 \\
\hline & Total tête & & 7 & 15 & 1 \\
\hline & Membres postérieurs & tibia & 1 & 13 & 1 \\
\hline & Total membres postérieurs & & 1 & 13 & 1 \\
\hline Total cerf & & & 8 & 28 & 2 \\
\hline Total os déterminés F84 & & & 34 & 1284 & - \\
\hline
\end{tabular}

Tabl. 2 - Distribution anatomique des restes de bœuf, de capriné et de cerf mis au jour dans la fosse 48 de la « rue des Castors » (exprimée en nombre et poids des restes, ainsi qu'en nombre minimum de parties du squelette).

Table 2 - Skeletal part frequency of cattle, sheep/goat and deer in slot pit 48, 'Rue des Castors' (expressed as number, weight and minimum number of elements). 
seule une vertèbre et six fragments de côtes ont été mis en évidence), ce dépôt correspond-il tout simplement à une fosse également utilisée comme dépotoir et qui aurait recueilli un rejet partiel lié au traitement boucher d'une carcasse de cerf?

\section{L'industrie lithique}

Seule la fosse 48 , située près de la grande maison danubienne, a livré neuf éléments lithiques dans son comblement supérieur. Il s'agit de petits éclats, d'une lame et de débris. Deux éclats sont des pièces esquillées (fig. 15).

Cette industrie s'intègre parfaitement dans le corpus lithique issu de l'occupation du Néolithique ancien attestée sur le site. Il s'agit vraisemblablement d'un rejet lié aux activités s'étant déroulées à l'extérieur des maisons. Les pièces esquillées ne semblent pas être en lien avec la fonction de la fosse, puisque d'autres outils du même type ont été retrouvés ailleurs sur le site.

\section{Les graines}

Le comblement médian de la fosse 32 , au nord, a livré douze graines de céréales, dont du blé amidonnier (déterminations F. Toulemonde). Néanmoins, la datation de ces graines suggère qu'elles sont intrusives (cf. infra) : elles témoigneraient de la mise en culture des terres à une époque ultérieure.

\section{Synthèse sur le mobilier des fosses à profil en $V$-Y}

Cinq des sept fosses livrent du mobilier archéologique. Celui-ci renvoie toujours, pour les éléments datant, au Néolithique ancien. Plusieurs contextes se démarquent et interrogent sur la taphonomie des fosses. La partie supérieure de la fosse 48 , isolée près d'une grande maison danubienne, a clairement servi de fosse-dépotoir aux occupants de la maison. À l'inverse, les autres fosses comportent plutôt du mobilier épars, sauf la fosse 19 qui ne livre que des os d'animaux sauvages, dont des os de cervidé en connexion anatomique retrouvés dans le fond. Le mobilier ne permet pas, à lui seul, de conclure quant à la fonction des fosses. La portion de cervidé de la fosse 19 est néanmoins intéressante, puisque la présence de restes d'animaux sauvages retrouvés en connexion anatomique dans le fond des fosses à profil en V-Y est souvent évoquée pour l'interprétation cynégétique (cf. supra). Cependant, dans notre cas, l'hypothèse d'un rejet de découpe au sein d'un contexte d'habitat est tout aussi viable.

\section{Datation et interprétation du dispositif}

L'un des os de cervidé issu du fond de la fosse 19 a fait l'objet d'une datation radiocarbone (Lyon-8444 GrA). La date obtenue est $6080 \pm 35 \mathrm{BP}$, soit entre 5193 et 4857 av. J.-C., qui peut être réduite entre 5065 et 4925 av. J.-C. ( $89 \%$ de probabilité). Cet assemblage est donc contemporain du village RRBP, qui livre des dates radiocarbones tout à fait similaires.

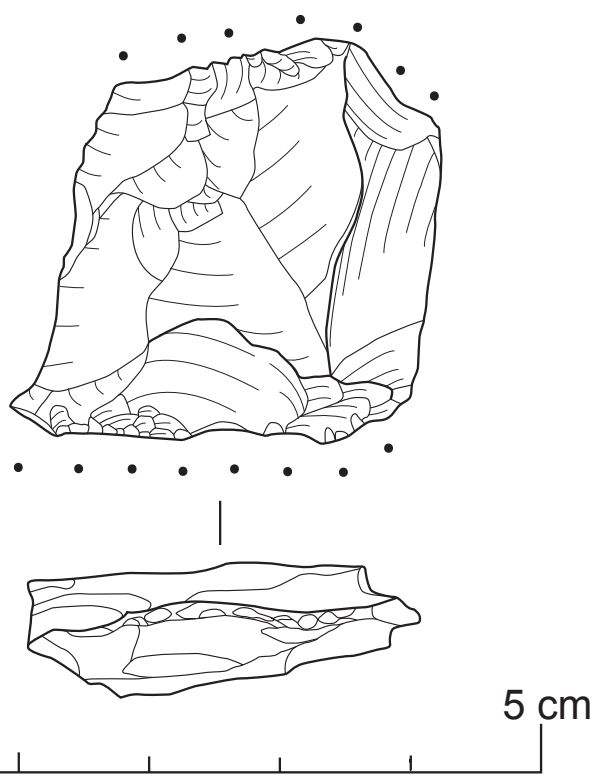

Fig. 15 - Pièce esquillée issue de la fosse 48 de la « rue des Castors » (dessin C. Carlier).

Fig. 15 - Splintered piece from slot pit 48, 'Rue des Castors' (drawing C. Carlier).

Une des graines de la fosse 32, qui se situe dans le même arc de cercle que la fosse 19, a également été datée par radiocarbone (Lyon-8710 GrA). La date, 5020 $\pm 35 \mathrm{BP}$, se situe ici au Néolithique moyen, entre 3944 et 3707 av. J.-C. Les graines sont issues du comblement médian de la fosse.

Les deux fosses étant dans un même dispositif en arc de cercle, il est fort probable qu'elles soient contemporaines. Les os en connexion anatomique, en fond de fosse, dans la couche supposée «fonctionnelle » si l'on retient l'hypothèse cynégétique, sont des éléments datant plus fiables que les graines qui pourraient être piégées lors du comblement de la fosse. La présence de mobilier du Néolithique ancien dans le comblement supérieur de plusieurs fosses vient d'ailleurs conforter cette datation.

On peut ainsi penser que ces fosses ont été creusées au Néolithique ancien, et que certaines étaient encore ouvertes (ou en cours de comblement) au Néolithique moyen. Ces fosses sont donc contemporaines du village RRBP, ou alors chronologiquement très proches. Elles sont, de fait, parmi les plus anciennes fosses de ce type connues à ce jour en Champagne-Ardenne (AchardCorompt et al., 2010).

Mais comment, alors, interpréter ces fosses si elles sont en relation avec le village Rubané?

Malgré la présence d'os de cervidé en connexion anatomique dans le fond d'une fosse, caractère couramment cité pour évoquer l'hypothèse cynégétique (cf. supra), celle-ci paraît contredite par la présence des habitations proches. En effet, la cohabitation entre des pièges de chasse et une communauté villageoise semble difficile, non seulement en raison des dangers induits pour les habitants et la faune domestique, mais également en 
raison de la faible chance de voir des animaux sauvages passer près des fosses. Plusieurs interprétations sont donc possibles.

La première est que les fosses seraient légèrement antérieures au village. Une implantation du Rubané Récent Champenois, phase chronologique antérieure au RRBP de Saint-Martin-sur-le-Pré, est attestée à $7 \mathrm{~km}$ sur le site de Juvigny « les Grands Traquiers » (Tappret et Villes, 1996). Les fosses pourraient ainsi être plus anciennes et avoir été rapidement rebouchées lors de l'installation des maisons au RRBP. La présence de mobilier du Rubané dans le sommet des fosses évoquerait bien un comblement rapide, et une des fosses a vraisemblablement servi de dépotoir à une des maisons. La portion de cervidé, en connexion anatomique au sein d'une fosse, corroborerait parfaitement une interprétation cynégétique. Cependant, cette hypothèse n'explique pas pourquoi le dispositif s'interrompt sur l'avant d'une des maisons danubiennes (qui n'existait alors pas si l'on suit le raisonnement), et la datation radiocarbone ne va pas en faveur d'une antériorité des fosses à profil en $\mathrm{V}-\mathrm{Y}$ sur le village, bien que ce matériau ne permette pas de discussions chronologiques fines en raison des effetspalier. L'argument le plus décisif est la datation des graines retrouvées dans une des fosses, qui indique que le dispositif était encore au moins partiellement ouvert au Néolithique moyen, ce qui vient donc contredire cette hypothèse.

Une autre hypothèse, qui conserverait l'interprétation cynégétique, serait que la batterie de fosses ait été construite après l'abandon du village. Cependant, l'absence de mobilier détritique dans le fond des fosses n'est alors pas expliquée. De même, la réutilisation en fosse-dépotoir du sommet d'une des fosses tend à contredire cette hypothèse, au final donc peu plausible.

La troisième hypothèse est que ces fosses seraient partie intégrante du village danubien. S'il n'est pas rare de retrouver des fosses à profil en $\mathrm{V}-\mathrm{Y}$ dans des villages du Rubané (Van de Velde, 1973; Jeunesse et Sainty, 1992), peu de ces sites livrent des fosses de ce type bien datées : elles peuvent ainsi être considérées comme diachroniques, ayant parfois piégé du mobilier antérieur et donc relever d'une autre occupation. Pourtant, il existe des exemples où, par leur organisation spatiale, des fosses à profil en V-Y sont incontestablement contemporaines de sites du Rubané : il s'agit des enceintes (fig. 16). En effet, sur les enceintes belges de Fexhe-le-Haut-Clocher et Rémicourt (Bosquet et al., 2007), une Schlitzgrube (fosse à profil en V-Y) est située au milieu de l'interruption du fossé d'enceinte du village, participant à un probable dispositif d'entrée. Ces fosses seraient, d'après les auteurs, la tranchée de fondation d'une petite palissade séparant l'entrée en deux couloirs distincts. Le site Rubané allemand de Langweiler 8 présente également, à une interruption de son fossé d'enceinte, deux fosses longues et étroites, profondes, avec un profil transversal en V (Boelicke, 1988, p. 407). La ressemblance avec les deux enceintes belges est telle qu'il semble évident que ces Schlitzgruben participent à un dispositif défensif de l'entrée, dont l'interprétation reste néanmoins complexe.

S'il n'y a pas de fossé d'enceinte connu à Saint-Martin-sur-le-Pré, en revanche, cinq fosses à profil en V-Y forment un dispositif en arc de cercle au nord des habitations, s'étendant hors emprise. Aucun indice ne semble suggérer la présence d'une palissade dans ces fosses; au contraire, tout porte à croire qu'elles aient été ouvertes lors de leur fonctionnement. La forte profondeur de ces fosses pourrait ainsi constituer une limite physique aux habitations. On peut tout à fait imaginer que la batterie de fosses, suivant un axe circulaire, vienne se refermer sur la partie est du village Rubané, en dehors de l'emprise fouillée.

Peut-on voir dans les fosses à profil en V-Y de SaintMartin-sur-le-Pré un dispositif défensif inédit, inspiré des enceintes belges et allemandes? La méconnaissance du plan complet du site ne permet malheureusement pas de discuter des limites physiques du village. Par son caractère atypique, cette interprétation reste à valider, et doit encore être confortée par la découverte d'autres exemples similaires.

Cette constatation invite à s'interroger sur les nombreuses découvertes de fosses à profil en V-Y sur les sites du Rubané rhénan (Van de Velde, 1973). En effet, sur ces sites, la contemporanéité stricto sensu des habitats néolithiques avec les fosses à profil en V-Y n'a jamais pu être démontrée et fait débat, malgré la présence de mobilier du Rubané dans certaines fosses comme à Hienheim. S'il existe probablement une diachronie entre ces structures sur plusieurs de ces sites, on peut néanmoins s'interroger sur la présence de dispositifs contemporains sur certains. Cette présomption rouvre, sans conclure, la question sur la fonction des fosses à profil en V-Y au sein des habitats du Rubané.

Le site de la « rue des Castors » à Saint-Martin-surle-Pré se singularise donc des nombreuses découvertes de fosses à profil en V-Y par la présence d'une batterie de fosses au sein même d'un habitat contemporain, ce qui est un cas totalement atypique en Champagne-Ardenne. Les fosses sont datées du Néolithique ancien : elles sont donc parmi les plus anciennes à ce jour. L'interprétation du dispositif est difficile, et l'hypothèse cynégétique n'est pas exclusive; celle d'un dispositif « défensif » au sens large peut également être avancée.

\section{CONCLUSION}

\footnotetext{
T es trois exemples présentés ici illustrent la diversité $\mathcal{L}$ des formes des batteries de fosses à profil en V-Y dans la Marne. Il est d'ailleurs fort probable que des découvertes futures illustrent d'autres formes de dispositifs de fosses. L'aire de chasse du Néolithique récent de Bezannes, au sud de Reims, avec un maillage de fosses très lâche sur une grande étendue (Bontrond et al., 2013), en est un exemple.
} 


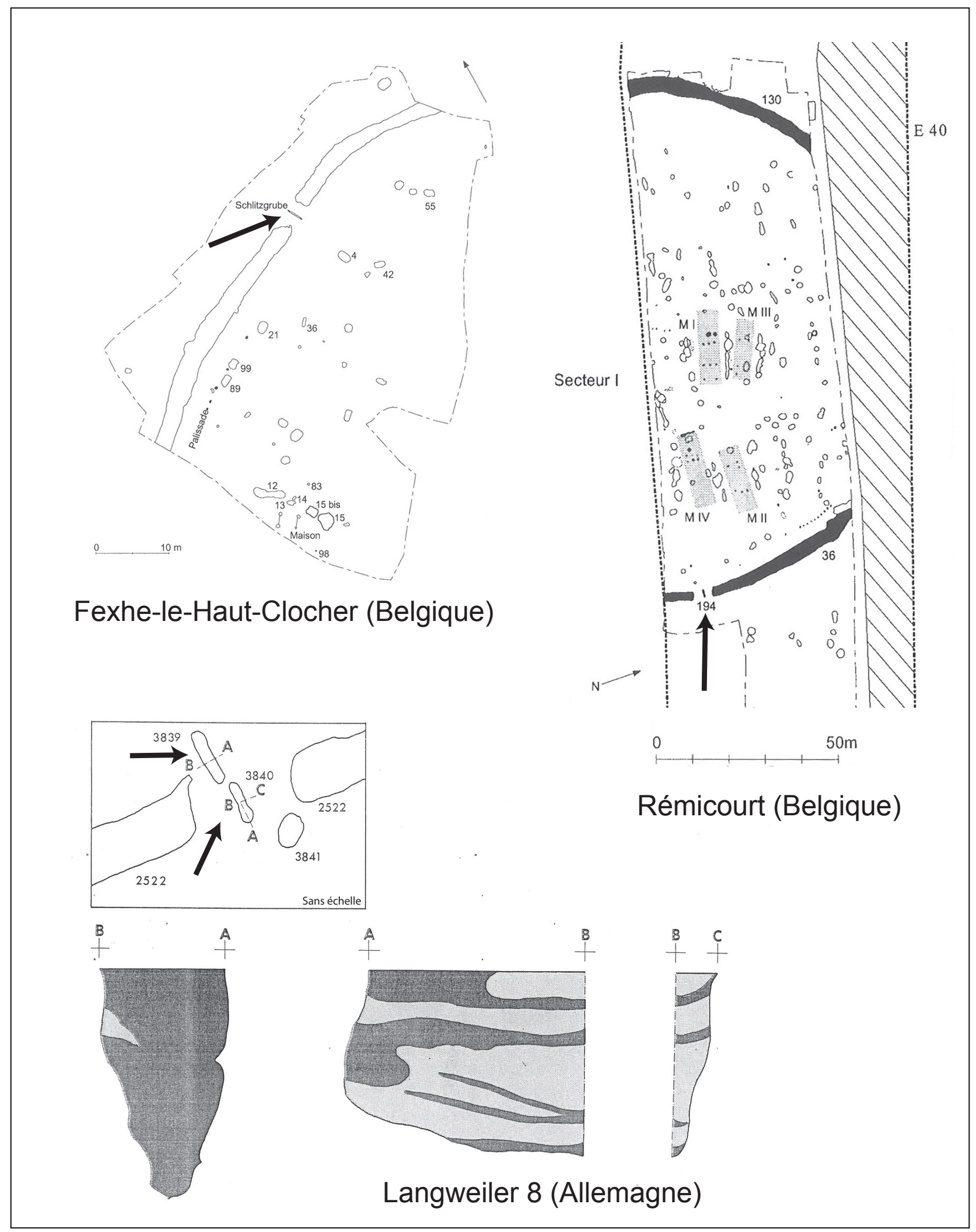

Fig. 16 - Schlitzgruben (fosses à profil en V-Y) dans des dispositifs d'entrée d'enceintes du Rubané (d'après Boelicke, 1988, et Bosquet et al., 2007).

Fig. 16 - Schlitzgruben in Linear Pottery enclosures (after Boelicke, 1988 and Bosquet et al., 2007). 
Afin de pouvoir comparer efficacement les fosses à profil en V-Y retrouvées dans le cadre de nos opérations de diagnostics et de fouilles, toutes celles sondées ont fait l'objet de sondages similaires et d'un même protocole d'échantillonnage. Trois prélèvements ont été réalisés dans chaque fosse : un dans le comblement supérieur, un dans le comblement médian et un dans celui du fond. Ces prélèvements ont ensuite été tamisés par flottation puis à maille fine, afin d'y rechercher toute trace anthropique ou naturelle pouvant aider à la détermination de la fonction des fosses : mobilier, graines, microfaune, restes malacologiques...

On peut ainsi tenter de rechercher des similitudes et des différences dans nos trois exemples :

- quel que soit le site, les fosses présentent des morphologies semblables, avec des profils transversaux en $\mathrm{V}$ ou $\mathrm{Y}$, et une profondeur située vers $-2 \mathrm{~m}$ depuis la surface du substrat;

- l'organisation spatiale des groupes de fosses est variable : groupe serré (« le Champ de Manœuvre »), ligne de fosse lâche barrant une pente (« les Écavés »), batterie resserrée en arc de cercle (« rue des Castors »);

- au sein d'un même dispositif, l'orientation des fosses est variable et ne semble soumise à aucune règle;

- la taille des fosses est également très variable, et ce au sein d'un même dispositif. Pour ceux de Bétheny, deux groupes de fosses semblent se distinguer par leurs dimensions. Le groupe des fosses « massives » est en revanche absent à Saint-Martin-sur-le-Pré. En l'état, il est difficile de dire si ces groupes ont une signification chronologique ou fonctionnelle;

- les comblements des fosses sont assez similaires : la moitié inférieure est très crayeuse, la moitié supérieure est très limoneuse. Parfois, un fin niveau limoneux marque le fond de la fosse. Pourtant, les tamisages systématiques livrent des résultats assez variés entre les sites : ils signalent que le mode de comblement des fosses peut varier d'un site à l'autre, voire même d'une fosse à l'autre (colluvionnement naturel, comblement volontaire, dépotoir...);

- à Bétheny, les sept fosses sondées sont quasiment vides de tout mobilier archéologique, si ce n'est quelques fragments de céramique en surface illustrant divers horizons chronologiques. À Saint-Martin-sur-le-Pré, cinq des sept fosses sondées livrent du mobilier archéologique (céramique, faune, industrie lithique, graines), dont des os de cervidé en connexion anatomique;
- dans deux cas, à Bétheny, les fosses sont loin de tout habitat présumé contemporain. En revanche, à SaintMartin-sur-le-Pré, les fosses sont intégrées au sein d'un habitat contemporain.

Ces comparaisons restent limitées, car les dispositifs de Bétheny ne sont encore que partiellement connus, notamment en ce qui concerne leurs bornes chronologiques. En effet, des exemples récents comme le site de Thillois « les Terres Soudées », à l'ouest de Reims (Bontrond et al., 2013) ont démontré que des fosses à profil en V-Y spatialement proches pouvaient montrer un écart chronologique important.

$\mathrm{Si}$, pour les deux dispositifs de Bétheny, l'hypothèse cynégétique peut être avancée sans problème, celle-ci est n'est cependant pas privilégiée pour la batterie de SaintMartin-sur-le-Pré. Les exemples des enceintes du Rubané belge et allemand, où des fosses à profil en V-Y sont intégrés dans le dispositif d'enceinte fossoyée des villages (cf. supra), permettent de proposer une autre hypothèse, plus ou moins liée à des dispositifs défensifs. Cette hypothèse demeure néanmoins malaisée en raison de la connaissance partielle du site de Saint-Martin-sur-le-Pré.

Ces trois sites illustrent bien les différentes formes que peuvent prendre les dispositifs de fosses à profil en V-Y. De nombreux éléments restent à comprendre, notamment sur la chronologie, le fonctionnement et la fonction même de ces batteries.

Les fosses à profil en V-Y du Rubané de Saint-Martin-sur-le-Pré sont, à ce jour, parmi les plus anciennes connues régionalement. Or, le « phénomène » des fosses à profil en V-Y s'étend, en Champagne-Ardenne, du Néolithique ancien à l'âge du Fer (Achard-Corompt et al., 2010). Il est tout à fait possible que la fonction cynégétique, présumée la plus courante en Champagne, ne soit qu'un dérivé d'une fonction première, au Néolithique ancien, qui n'a ensuite pas persisté au sein des habitats.

Ces exemples montrent bien que la compréhension de ces fosses, souvent retrouvées seules et isolées, ne peut être appréhendée que par des recherches larges, tant sur le plan spatial que chronologique. Les fouilles menées dans le futur devraient, nous l'espérons, alimenter encore largement ces problématiques.

\section{NOTE}

(1) Remerciements à M. Ilett pour la traduction du résumé.

\section{RÉFÉRENCES BIBLIOGRAPHIQUES}

Achard-Corompt G., Achard-Corompt N., Desbrosse V., Auxiette G., Fechner K., Moreau C., Peltier V., Riquier V., Vanmoerkerke J. (2010) - Chasse, culte ou artisanat? Premiers résultats du projet de recherche relatif aux fosses à profil « en V, Y, W », Bulletin de la Société préhistorique française, 107, 3, p. 588-591.

Achard-Corompt N., Auxiette G., Fechner K., Riquier V., VAnmoerkerke J. (2013) - Bilan du programme de recherche : fosses à profil en $\mathrm{V}, \mathrm{W}, \mathrm{Y}$ et autres en Champagne-Ardenne, in $\mathrm{N}$. Achard-Corompt et V. Riquier (dir.), Chasse, culte ou artisanat? Les fosses «à profil en $Y-V-W$ ». Structures énigmatiques et récurrentes du Néolithique aux âges des Métaux en France et alentour, actes de la table ronde (Châlons-en-Champagne, 15-16 novembre 2010), Dijon, Société archéologique de l'Est (Supplément à la Revue archéologique de l'Est, 33), p. 11-81. 
Boelicke U. (1988) - Das Erdwek, in U. Boelick et R. W. Aniol (dir.), Der bandkeramische Siedlungsplatz Langweiler 8, Bonn, Rheinland-Verlag (Beiträge zur neolithischen Besiedlung der Aldenhovener Platte, 3 ; Rheinische Ausgrabungen, 28), p. 395-427.

Bontrond R., Bündgen S., Charles L., Garmond N., Poupon F. (2013) - Deux ensembles de fosses à profil en «V-Y » de la périphérie rémoise : Bezannes et Thillois (Marne), in N. Achard-Corompt et V. Riquier (dir.), Chasse, culte ou artisanat? Les fosses "à profil en $Y-V-W »$. Structures énigmatiques et récurrentes du Néolithique aux âges des Métaux en France et alentour, actes de la table ronde (Châlons-en-Champagne, 15-16 novembre 2010), Dijon, Société archéologique de l'Est (Supplément à la Revue archéologique de l'Est, 33), p. 93-108.

Bosquet D., Goffioul C., Preud'homme D. (2007) - Les enceintes rubanées de Belgique : apports des fouilles récentes, in O. Agogué, D. Leroy et C. Verjux (dir.), Camps, enceintes et structures d'habitat néolithiques en France septentrionale, actes du $24^{\mathrm{e}}$ Colloque interrégional sur le Néolithique, (Orléans, 19-21 novembre 1999), Tours, FERACF (Supplément à la Revue archéologique du Centre de la France, 27), p. 5-20.

Constantin C., Ilett M. (1997) - Une étape finale dans le Rubané récent du Bassin parisien, in C. Jeunesse (dir.), Le Néolithique danubien et ses marges entre Rhin et Seine, actes du $22^{\mathrm{e}}$ Colloque interrégional sur le Néolithique, (Strasbourg, 27-29 octobre 1995), Strasbourg, Association pour la promotion de la recherche archéologique en Alsace (Monographies d'archéologie alsacienne, 3), p. 281-300.

Desbrosse V. (2011) - Les fosses à profil en U, V et Y à l'emplacement de la distillerie Cristanol à Bazancourt-Pomacle : relations spatiales et chronologiques dans le paysage, Bulletin de la Société archéologique champenoise, 104, 2, p. 11-25.

FrIEDERICH S. (à paraître) - Schlitzgrüben. Ein Tierfallensystem, in in N. Achard-Corompt et V. Riquier (dir.), Chasse, culte ou artisanat? Les fosses « à profil en $Y-V-W »$. Structures énigmatiques et récurrentes du Néolithique aux âges des Métaux en France et alentour, actes de la table ronde (Châlons-en-Champagne, 15-16 novembre 2010), Dijon, Société archéologique de l'Est (Supplément à la Revue archéologique de l'Est, 33), p. 229-243.

Garmond N., dir. (2012) - Saint-Martin-sur-le-Pré « rue des Castors », phases 1 et 2, Marne, Champagne-Ardenne, 1. Le village du Néolithique ancien, rapport final d'opération, Reims Métropole, service régional de l'Archéologie de Champagne-Ardenne, Reims, 333 p.

Garmond N., Arnaud M. (2011) - Bétheny, Reims «la Husselle ", Marne, Champagne-Ardenne, rapport de diagnostic archéologique, Reims Métropole, service régional de l'Archéologie de Champagne-Ardenne, Reims, 77 p.

Garmond N., Arnaud M., Bouquin D., Brives A.-L., Huart L., Lebouvier A. (2011) - Bétheny «les Ecavés », tranche 1, Marne, Champagne-Ardenne, rapport de diagnostic archéologique, Reims Métropole, service régional de l'Archéologie de Champagne-Ardenne, Reims, 118 p.
Garmond N., Binder S., Bonnardin S., Bouquin D., Hamon C., Lefevre C., Poupon F. (2012) - L'habitat Rubané de Saint-Martin-sur-le-Pré « Rue des Castors » (Marne). Premiers résultats, Internéo 9 (journée d'information du 17 novembre 2012), p. 35-47.

Garmond N., Huart L., Laudrin F. (2012) - Bétheny "les Ecavés ", tranche 2, Marne, Champagne-Ardenne, rapport de diagnostic archéologique, Reims Métropole, service régional de l'Archéologie de Champagne-Ardenne, Reims, $132 \mathrm{p}$.

Jeunesse C., Sainty J. (1992) - L'habitat néolithique ancien du site d'Hensisheim « Ratfeld» (Haut-Rhin), Cahiers de l'Association pour la promotion de la recherche archéologique en Alsace, 8, 1, p. 95-146.

Marcigny C., Riquier V. (2009) - Les sites à « fosses en $\mathrm{V}-\mathrm{Y} »$. Emergence d'une problématique et d'un réseau au niveau national, Archéopages, 25, p. 70-75

PARET O. (1910) - Das Steinzeitdorf bei Monrepos, Fundberichte aus Schwaben, 18, p. 6-8.

Saurel M., Bocquillon H., Duda D., Turé I. (2006) - Recy (Marne) "Parc industriel de référence », tranche 1, rapport de diagnostic archéologique, service régional de l'Archéologie de Champagne-Ardenne, Châlons-en-Champagne.

TAprret E., Villes A. (1996) - Contribution de la Champagne à l'étude du Néolithique ancien, in La Bougogne entre les bassins rhénan, rhodanien et parisien. Carrefour ou frontières? actes du $18^{\mathrm{e}}$ Colloque interrégional sur le Néolithique (Dijon, octobre 1991), Dijon, Société archéologique de l'Est (Supplément à la Revue Archéologique de l'Est, 14), p. 175-256.

Van de Velde P. (1973) - Rituals, Skins and Homer: The Danubian « Tan-Pits », Analecta Praehistorica Leidensia, 6, p. 35-50.

Nicolas GARMOND

UMR 8215 « Trajectoires » responsable scientifique d'opération Service archéologique de Reims Métropole 6, rue du Val Clair, 51100 Reims nicolas.garmond@reimsmetropole.fr

Sophie BINDER céramologue

69, rue Ponsardin, 51100 Reims sophiebinder@free.fr

Frédéric Poupon archéozoologue

UMR 7324 « CITERES-LAT » Service archéologique de Reims Métropole 6, rue du Val Clair, 51100 Reims frederic.poupon@reimsmetropole.fr 